\title{
Evaluation of fast atmospheric dispersion models in a regular street network
}

Article

Accepted Version

Hertwig, D., Soulhac, L., Fuka, V., Auerswald, T., Carpentieri, M., Hayden, P., Robins, A., Xie, Z.-T. and Coceal, O. (2018) Evaluation of fast atmospheric dispersion models in a regular street network. Environmental Fluid Mechanics, 18 (4). pp. 1007-1044. ISSN 1567-7419 doi: https://doi.org/10.1007/s10652-018-9587-7 Available at https://centaur.reading.ac.uk/75854/

It is advisable to refer to the publisher's version if you intend to cite from the work. See Guidance on citing.

To link to this article DOI: http://dx.doi.org/10.1007/s10652-018-9587-7

Publisher: Springer

All outputs in CentAUR are protected by Intellectual Property Rights law, including copyright law. Copyright and IPR is retained by the creators or other copyright holders. Terms and conditions for use of this material are defined in the End User Agreement.

www.reading.ac.uk/centaur 
Central Archive at the University of Reading

Reading's research outputs online 


\title{
Evaluation of fast atmospheric dispersion models in a regular street network
}

\author{
Denise Hertwig · Lionel Soulhac · Vladimír \\ Fuka - Torsten Auerswald - Matteo Carpentieri . \\ Paul Hayden · Alan Robins · Zheng-Tong Xie · \\ Omduth Coceal
}

Received: date / Accepted: date

\begin{abstract}
The need to balance computational speed and simulation accuracy is a key challenge in designing atmospheric dispersion models that can be used in scenarios where near real-time hazard predictions are needed. This challenge is aggravated in cities, where models need to have some degree of building-awareness, alongside the ability to capture effects of dominant urban flow processes. We use a combination of high-resolution large-eddy simulation (LES) and wind-tunnel data of flow and dispersion in an idealised, equal-height urban canopy to highlight important dispersion processes and evaluate how these are reproduced by representatives of the most prevalent modelling approaches: (i) a Gaussian plume model, (ii) a Lagrangian stochastic model and (iii) street-network dispersion models. Concentration data from the LES, validated against the wind-tunnel data, were averaged over the volumes of streets in order to provide a high-fidelity reference suitable for evaluating the different models on the same footing. For the particular combination of forcing wind
\end{abstract}

D. Hertwig, T. Auerswald, O. Coceal

Department of Meteorology, University of Reading

P.O. Box 243, Reading, RG6 6BB, UK

E-mail: d.hertwig@ reading.ac.uk

Tel.: +44-118-378-6721

Fax: +44-118-378-8905

L. Soulhac

Laboratoire de Mécanique des Fluids et d'Acoustique

École Centrale de Lyon, 69134 Ecully, France

V. Fuka, Z-T. Xie

Faculty of Engineering and the Environment

University of Southampton, Southampton, SO17 1BJ, UK

M. Carpentieri, P. Hayden, A. Robins

Enflo, Department of Mechanical Engineering Sciences

University of Surrey, Guildford, GU2 7XH, UK

P. Hayden, O. Coceal

National Centre for Atmospheric Science, UK 
direction and source location studied here, the strongest deviations from the LES reference were associated with mean over-predictions of concentrations by approximately a factor of 2 and with a relative scatter larger than a factor of 4 of the mean, corresponding to cases where the mean plume centreline also deviated significantly from the LES. This was linked to low accuracy of the underlying flow models/parameters that resulted in a misrepresentation of pollutant channelling along streets and of the uneven plume branching observed in intersections. The agreement of model predictions with the LES (which explicitly resolves the turbulent flow and dispersion processes) greatly improved by increasing the accuracy of building-induced modifications of the driving flow field. When provided with a limited set of representative velocity parameters, the comparatively simple street-network models performed equally well or better compared to the Lagrangian model run on full $3 \mathrm{D}$ wind fields. The study showed that street-network models capture the dominant building-induced dispersion processes in the canopy layer through parametrisations of horizontal advection and vertical exchange processes at scales of practical interest. At the same time, computational costs and computing times associated with the network approach are ideally suited for emergency-response applications.

Keywords Pollutant dispersion · Urban environment - Street-network model · Gaussian plume model $\cdot$ Lagrangian stochastic model $\cdot$ Model inter-comparison

\section{Introduction}

In the event of hazardous materials being released into the atmosphere, either by accident or intentionally, dispersion models are key to coordinate actions to avoid or mitigate impacts on human health $[11,31,63]$. Emergency response dispersion models are applied both proactively, e.g. to assess exposure risks and vulnerability of sensitive public structures, and reactively as part of emergency management protocols and decision making frameworks [34]. Principal areas of application can be grouped into (i) planning (pre-incident), (ii) response (mid-incident) and (iii) analysis/evaluation (post-incident).

In general, an emergency response dispersion model needs to have short latency times to enable timely actions (fast), it should make low demands on computational resources required, be easy to use and fast to set up (cheap) and the results produced should be accurate and interpretable in an unambiguous way. Figure 1 illustrates these requirements in terms of a 'feasibility triangle'. The dilemma faced in emergency response modelling is that once two of these requirements are met, fulfilling the remaining third becomes a challenge. For example, in order to make accurate calculations quickly, computational requirements and costs are high; fast and cheap models have accuracy limitations; accurate and computationally expensive models require long run times. Hence, as long as computational resources remain limited, model developers are tasked with finding an optimal balance between these requirements.

\subsection{Challenges in urban areas}

High population density and limited evacuation options increase human exposure risks in cities, making them particularly vulnerable to hazards from air-borne contaminants. Quality requirements on urban dispersion models hence are high. The challenge to balance speed and accuracy is exacerbated since urban dispersion models need to have some degree of building-awareness, alongside the ability to capture complex effects of urban flow patterns 


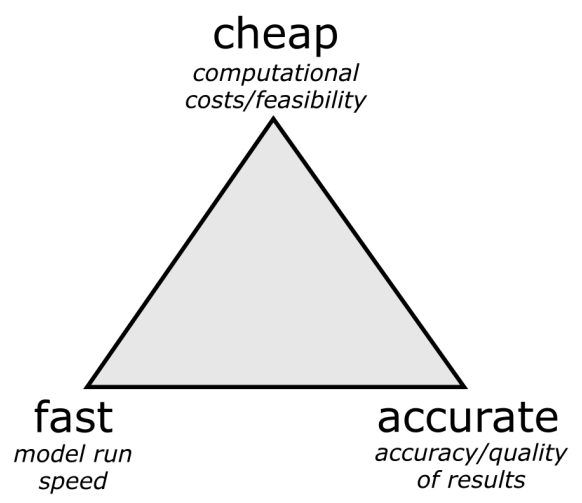

Fig. 1: 'Feasibility triangle’ for emergency response dispersion modelling.

on the dispersion process $[18,5]$. Numerous field, laboratory and numerical experiments of the past have shown that the impact of buildings on pollutant dispersion is significant, particularly in the near-field close to the source $[27,14,50,82,79]$. Due to building-induced flow effects like channelling, branching in intersections, wake recirculation or vortex shedding at roof and building corners, plume dispersion within the urban canopy layer (UCL) is distinctively different from dispersion well above the roughness sublayer. Building arrangements and street layouts uniquely determine this so-called topological component of urban dispersion. Material can travel significant distances upstream of the source if trapped in recirculating wind regimes [82]. Localised trapping of pollutants in building wakes can create secondary sources whose emission characteristics are governed by local flow properties and can vastly differ from those of the primary source [5]. In addition, strong variations in building heights can result in significant asymmetries of the vertical plume structure with material being lifted out of the canopy layer, resulting in a shift of the effective source height $[44,45,15]$.

Computational fluid dynamics (CFD) approaches like Reynolds-Averaged Navier-Stokes (RANS) modelling or large-eddy simulation (LES), and to a lesser extent wind-tunnel experiments, can deliver detailed information about flow and dispersion processes in built environments [79]. While CFD models can be specifically designed for emergency response planning and preparation $[44,30]$, associated computing times currently are too long for operational use during emergency events [80].

Instead, simpler model formulations are needed that represent processes relevant for the scenario through suitable parametrisations and ideally can also be operated in inverse mode for source detection. Approaches for fast urban dispersion modelling are discussed below. For an overview of urban dispersion models see e.g. Andronopoulos et al. [2].

\subsection{Options for fast urban dispersion modelling}

Urban emergency response models are primarily applied to the dispersion of air-borne substances from localised releases from a limited number of sources. Typical time scales of interest range from seconds to a few hours and length scales from streets to city extents. Models currently used for fast dispersion simulations differ significantly in the way they 
Table 1: Characteristics of different modelling approaches for dispersion from localised releases in cities.

\begin{tabular}{|c|c|c|c|}
\hline Type & Dispersion & Flow & Buildings \\
\hline CFD & $\begin{array}{l}\text { Eulerian or } \\
\text { particle tracking }\end{array}$ & $\begin{array}{l}\text { internally computed mean } \\
\text { or turbulent velocities }\end{array}$ & explicit \\
\hline Gaussian & $\begin{array}{l}\text { analytical, } \\
\text { empirical }\end{array}$ & $\begin{array}{l}\text { mean plume advection } \\
\text { velocity (prescribed or modelled) }\end{array}$ & implicit \\
\hline Lagrangian & particle tracking & $\begin{array}{l}\text { externally computed mean flow, } \\
\text { turbulent variances, } \\
\text { Lagrangian time scales }\end{array}$ & explicit / implicit \\
\hline Street-network & flux balance & $\begin{array}{l}\text { mean horizontal } \\
\text { advection velocities, } \\
\text { vertical exchange } \\
\text { velocities (prescribed or modelled) }\end{array}$ & street topology \\
\hline Hybrid & nomographs & externally computed flow statistics & explicit / implicit \\
\hline
\end{tabular}

represent the built environment and account for urban flow and dispersion processes, as summarised in Tab. 1.

Here, the comparatively expensive flow-resolving and building-representing CFD solutions are included as a reference. At the other end of the complexity spectrum we find the widespread class of Gaussian dispersion models. Gaussian plume models are based on an empirical-analytical representation of the downwind concentration spread, with the plume shape being determined through empirically defined concentration standard deviations in lateral and vertical direction. In its simplest configuration, this model needs as input only an estimate of the mean velocity along the plume trajectory $U_{p}$. Gaussian plume models have been extensively tested and advanced model versions include parametrisations of effects of atmospheric stratification, complex terrain or built environments. The US EPA's model AERMOD [25] takes into account urban effects through enhanced turbulence levels relative to rural areas and includes a module (PRIME) that accounts for plume downwash in the wake of single buildings. The UK's ADMS model [22] in its urban version ADMS-urban [51] uses the Operational Street Pollution Model (OSPM) [46,8] to model street canyon effects. Flow and dispersion effects around isolated buildings are modelled in the ADMSBUILD module [58].

With Gaussian puff models short-duration, non-steady-state releases are modelled by tracking the path of individual pollutant clouds in the flow (in a Lagrangian sense). Within the Urban Dispersion Model (UDM) [16] bulk effects of single buildings, building clusters, or entire cities on puff trajectories are parametrised. This distinguishes UDM from Lagrangian Gaussian puff models like RIMPUFF [77], SCIPUFF [75, 1] or CALPUFF [62], which are used on the regional/meso-scale and treat cities in a bulk way as an urban roughness. All of these models are integral components of several national and multi-national emergency response support systems.

Lagrangian stochastic dispersion models compute trajectories of computational particles in $3 \mathrm{D}$ wind fields using random-walk methods to represent the stochastic component of the dispersion process. Compared to typical Gaussian or building-resolving CFD models, Lagrangian models can be applied to problems ranging from local to global scales. Usually, Lagrangian models are run off-line on wind fields supplied by diagnostic or prognostic models, e.g. numerical weather prediction models for applications from regional to global scales 
and CFD or diagnostic wind models for urban-scale problems. Well-known representatives of off-line Lagrangian models used operationally across scales are the UK Met Office's NAME model [49] or NOAA's HYSPLIT model [74]. Examples of Lagrangian randomwalk dispersion models applied in built environments are LANL's QUIC-PLUME model [81] and Micro-Swift-Spray (MSS) [78]. In both QUIC-PLUME and MSS flow information is provided by built-in wind models based on empirical-diagnostic representations of building-induced flow effects.

Street-network models are a comparatively recent addition to the family of urban dispersion models, first brought forward by Soulhac [67]. Here, urban areas are represented through a network of connected boxes, covering street canyons and intersections, and canopylayer dispersion is simulated by parametrising concentration fluxes between these boxes $[38,6]$. While not representing buildings explicitly, the model is directly aware of the street topology of the city. Like Gaussian dispersion models, street-network models require only few flow specifications, which can be either imported from an external flow simulation or obtained through suitable parametrisations. The only street-network models currently used operationally are the SIRANE [71,72] model and its unsteady version SIRANERISK [69], which both contain built-in flow parametrisations.

A further approach was introduced by the US Naval Research Laboratory with the hybrid plume dispersion model CT-Analyst [11]. This model produces real-time urban concentration predictions by interrogating databases containing possible contaminant pathways for the release scenario [10]. These pathways have to be calculated in advance from detailed 3D flow simulations with building-resolving LES for different ambient wind directions and atmospheric conditions.

\subsection{Aims of this study}

In this study we aim to document strengths and limitations of prevalent dispersion modelling approaches with regard to the physical processes they capture. We choose the canonical test case of a localised release in an array of cuboidal buildings with oblique wind forcing. The models considered here are: (1) a baseline Gaussian plume dispersion model, (2) a Lagrangian stochastic plume model driven by $3 \mathrm{D}$ wind fields from models of varying complexity, and (3) two street-network dispersion models. While in some cases well-known representatives of these categories are used, the chief aim of this study is to highlight differences in modelling frameworks rather than ranking particular models. The fact that these approaches represent urban dispersion processes through vastly different modelling helps to identify which of these processes are of importance. By including the comparatively new street-network modelling approach as an alternative to traditional approaches and putting the focus on near-field dispersion patterns, this study adds further insight to previous model inter-comparison studies [57,56,60,41,3,4].

Furthermore we aim (i) to assess where in the hierarchy of fast dispersion modelling approaches the street-network model is situated by assessing its performance against more established methods, (ii) to investigate the effect of the accuracy and level of detail of the flow representation in the different types of models, and hence (iii) to gain insight into how existing parametrisations in such models could be improved. The dispersion characteristics are analysed based on datasets from boundary-layer wind-tunnel measurements and high-resolution large-eddy simulation of plume dispersion in an idealised urban environment comprised of a regular uniform array of cuboidal buildings. The performance of the LES has previously been validated successfully regarding its representation of flow and 
dispersion processes for this geometry based on the wind-tunnel experiments [23,32]. We extend this evaluation with a focus on particular aspects of the dispersion characteristics and then use the LES as a reference to establish differences between the output from the simpler models, averaged over the volumes of streets to reflect a common representation that matches the output from street-network models.

This work is part of the DIPLOS project (DIsPersion of LOcalised releases in Street networks; www.diplos.org) that aimed to improve parametrisations of dispersion processes in cities through a better understanding of time-dependent canopy-layer flow processes. Details about the test case and the reference data are presented in Sect. 2, followed by a brief introduction of the dispersion models used (Sect. 3). Flow and dispersion characteristics are discussed in Sect. 4, followed by an overview of the model inter-comparison study in Sect. 5. Conclusions are presented in Sect. 6.

\section{Reference experiment and simulation}

\subsection{Urban test geometry}

Given the interest in hazard modelling in populous areas, we are particularly interested in a geometric regime characteristic of city centres, and more specifically of European cities. To a fair degree of realism, such urban environments may be approximated by large rectangular blocks sufficiently close together as to produce a measure of decoupling between canopylayer flow and the external boundary layer. This means that street-canyon flow is fully developed and the city centre may be viewed as a network of streets joined at intersections [6]. With this in mind, the DIPLOS test geometry was designed as an array of aligned rectangular buildings of uniform height $H$ and street width $W=H$ (Fig. 2a), corresponding to the so-called skimming-flow regime. Each building has a dimension of $1 H \times 2 H \times 1 H$ in $x, y$ and $z$. In contrast to canonical cube-array settings, the rectangular buildings of the DIPLOS array introduce a geometrical asymmetry that is more typical of actual street topologies. A similar set-up is that of the well-studied MUST field-experiment configuration consisting of an aligned array of shipping containers [9]. However, with a canyon aspect ratio of $H / W=1$ the DIPLOS array produces more pronounced street-canyon flow behaviour typical for skimming-flow regimes compared to the rather 'open' MUST geometry with $H / W \simeq 0.2$ [61]. The plan area density, defined as the ratio of the area covered by buildings to the total area, has a value of $\lambda_{p}=0.33$ irrespective of model orientation. The frontal area density (ratio between the silhouette area of the buildings to the total plan area) is $\lambda_{f}=0.35$ for a model orientation of $-45^{\circ}$ that is investigated in this study.

\subsection{Reference data}

\subsubsection{Wind-tunnel experiment}

Flow and dispersion experiments under neutral stratification conditions were conducted in the Enflo laboratory at the University of Surrey. The open-return boundary-layer windtunnel used in this study has a $20 \mathrm{~m}$ test section and a cross section of $3.5 \mathrm{~m} \times 1.5 \mathrm{~m}$. The urban scale-model consisted of a regular array of $14 \times 21$ rows of wooden blocks of height $H=70 \mathrm{~mm}$. The model was mounted on a turntable whose centre was located about $14 \mathrm{~m}$ downstream of the test-section entrance. In this study we focus on a model orientation 
of $-45^{\circ}$ to the approaching boundary-layer flow; i.e. none of the streets are aligned with the inflow direction. As can be seen in Fig. 2a, in this set-up the corners of the model array were slightly curtailed in order to fit the array into the tunnel. In the flow development section upstream of the model, a fully-rough boundary-layer flow was modelled by the use of $1.26 \mathrm{~m}$ tall vorticity generators (Irwin spires) placed at the tunnel entrance and a staggered array of roughness elements covering the tunnel floor, resulting in a boundary-layer depth of about $14 \mathrm{H}$. Measurements within the model took place sufficiently far away from the leading edge of the model where the mean flow in any repeating unit as shown in Fig. 2b was verified to be independent of the location within the centre of the array. The tunnel freestream velocity of $U_{e}=2 \mathrm{~m} \mathrm{~s}^{-1}$ was constantly monitored downwind of the model by two reference ultrasonic anemometers positioned at a height of approximately $14.5 \mathrm{H}$. Castro et al. [23] estimate the friction velocity above the array to be $u_{*} / U_{e}=0.0891$, i.e. $0.178 \mathrm{~m} \mathrm{~s}^{-1}$. The roughness length, $z_{0}$, was determined by a fit of the data to the logarithmic wind profile using a von Kármán constant of $\kappa=0.39$ and a zero-plane displacement height derived from the LES (detail provided in Sect. 2.2.2). This resulted in a value of $z_{0} / H=0.039$.

Plume dispersion from a ground source was realised through the continuous release of a passive trace gas, for which a sufficiently diluted propane-air mixture was used to eliminate buoyancy effects. The source had an internal diameter of $20 \mathrm{~mm}$ (i.e. approx. $0.29 \mathrm{H}$ ) and was located in the middle of one of the long streets close to the centre of the model (Fig. 2c). The relatively large source diameter in combination with a very low flow rate of $Q=1.41 \mathrm{~min}^{-1}$ minimised momentum effects associated with the release through the source area and tests showed that residual effects are only non-negligible very close to the release location.

Point-wise concentration time-series were recorded using a Cambustion fast flame ionisation detector (FFID), capable of measuring hydrocarbon concentration fluctuations at a frequency of $200 \mathrm{~Hz}$. Velocity measurements were conducted with a two-component Dantec LDA system with a focal length of $160 \mathrm{~mm}$ providing a measuring volume with a diameter of $0.074 \mathrm{~mm}$ and a length of $1.57 \mathrm{~mm}$. The flow was seeded with micron-sized sugar particles at a sufficient level to attain flow sampling rates around $100 \mathrm{~Hz}$. All data were acquired over a measurement duration of $2.5 \mathrm{~min}$. The measurement sites analysed in this study are shown in Fig. 2c. Horizontal transects for the mapping of the plume footprint were conducted at nominal heights of $z / H=0.5$ and 1.5 , measuring concentrations and horizontal flow components. As discussed by Castro et al. [23], positional errors of the probes in the horizontal plane relative to the height of the buildings were corrected for in a post-processing step. In the data analysed here, the height range for individual measurement points was $0.44 \mathrm{H}$ to $0.54 \mathrm{H}$ and $1.44 \mathrm{H}$ to $1.54 \mathrm{H}$, respectively. Further uncertainties have to be expected with regard to the accuracy of the turntable orientation. Particularly for cases where the array is aligned with the approach flow, slight offsets can lead to strong differences in dispersion features as discussed by Fuka et al. [32]. Vertical profiles of paired velocity (all components) and concentration signals are available over a height range of $z / H=0.29$ to 5 . Scalar fluxes were measured using a laser Doppler anemometer (LDA), acquiring velocity signals, together with the concentrations signals measured by the FFID. For the vertical turbulent concentration fluxes, $\overline{c^{\prime} w^{\prime}}$, analysed here the FFID probe had a constant positional offset to the LDA measuring volume of $+2 \mathrm{~mm}$ in $x$ direction ( $3 \%$ of $H$ ) and $-5 \mathrm{~mm}$ in $y$ direction ( $7 \%$ of $H$ ). The implications of these spatial offsets obviously depend on local velocity and concentration gradients and will be discussed in the analysis of the data. Details of the fluxmeasurement set-up and associated uncertainties are described by Carpentieri et al. $[19,20]$ for similar experiments conducted in another city geometry. 


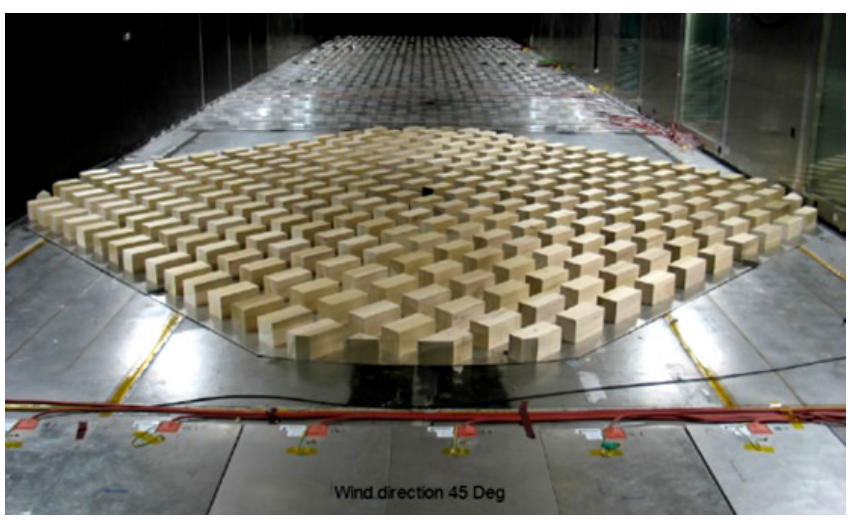

(a)

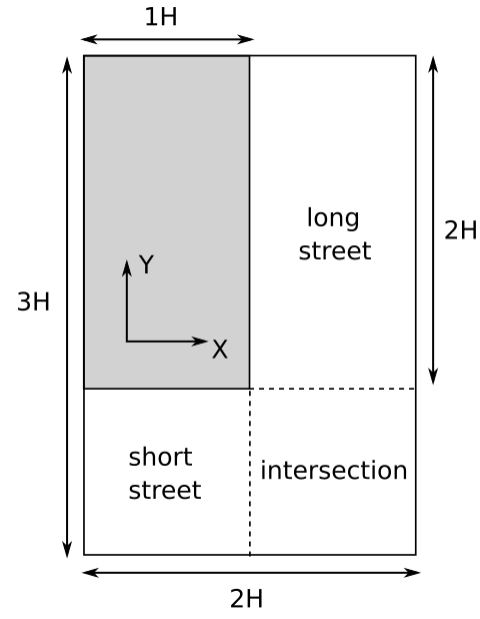

(b)

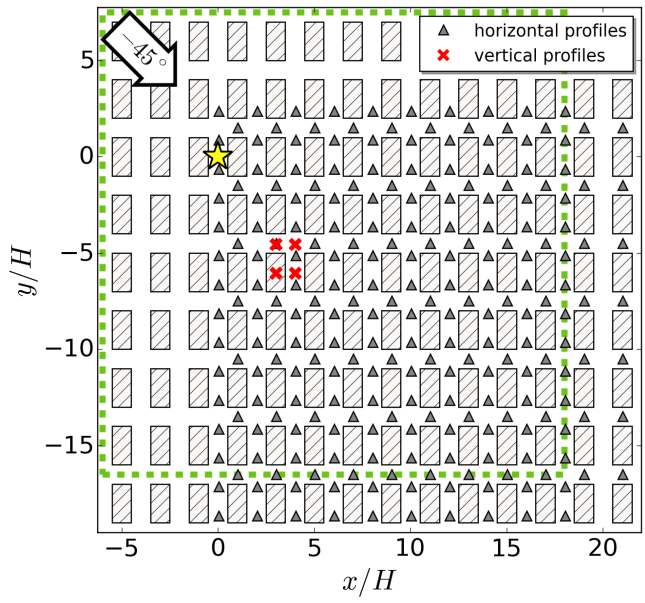

(c)

Fig. 2: (a) Upstream view of the DIPLOS array mounted in the Enflo wind tunnel for a model orientation of $-45^{\circ}$. Floor roughness elements and vorticity generators used to produce a thick approach-flow boundary-layer can be seen upstream of the array. (b) Plan-view of the repeating unit of the array, including the $1 H \times 2 H$ building (grey shading), long and short streets and an intersection. (c) Plan-view of a cut-out of the DIPLOS wind-tunnel array. The ground source is located at $x / H=y / H=0$ (star symbol). Wind-tunnel measurement locations (triangles: horizontal profiles; crosses: vertical profiles) and the horizontal extent of the $24 H \times 24 H$ LES computational domain (dashed square) are indicated. In (b) and (c) a coordinate system aligned with the streets is used (short streets along $x$ direction; long streets along $y$ direction). 


\subsubsection{Large-eddy simulation}

LES of flow and scalar dispersion was carried out at the University of Southampton using the open-source CFD package OpenFOAM (v2.1) and a mixed time-scale eddy-viscosity subgrid model [47]. The DIPLOS test case was simulated in a computational domain of size $24 H \times 24 H \times 12 H$ using a uniform Cartesian grid with a resolution of $\Delta=H / 16$.

As in the wind-tunnel experiment, passive, non-buoyant scalars were released continuously from a localised ground-source. The quasi-circular area source comprised 12 grid cells resulting in an effective source diameter of $0.244 \mathrm{H}$, which is comparable to the experimental set-up. No-slip conditions were imposed on all solid surfaces. With a stress-free boundary condition at the top of the domain and periodic boundary conditions in horizontal directions, the case was effectively realised as a planar channel flow. For the concentration fields, sponge layers were implemented at the outlet boundaries to prevent material from re-entering the domain through the inlet boundaries as part of the flow recycling process. Flow and concentration statistics were obtained over averaging periods of $1000 \mathrm{~T}$, where $T=H / u_{*}$ is the eddy-turnover time and $u_{*}$ is the friction velocity. The flow simulation was started from an initial field of resolution $\Delta=H / 16$, which was interpolated from a fullydeveloped precursor simulation of reduced resolution $(\Delta=H / 8)$. A spin-up time of $100 T$ was allowed before starting the pollutant release. Concentration statistics were computed by ensemble-averaging the time-averaged statistics derived from four independent realisations of the dispersion scenario.

As documented by Castro et al. [23] and Fuka et al. [32], for the same computational set-up the flow and dispersion simulations in a smaller domain $(12 \mathrm{H} \times 12 \mathrm{H} \times 12 \mathrm{H})$ were successfully validated against wind-tunnel measurements and data from direct numerical simulations (DNS) based on mean flow and turbulence statistics. Detailed descriptions of the flow simulations and the numerical techniques involved can be found in these publications. The friction velocity derived from the LES for the test case presented here (larger domain of $24 H \times 24 H \times 12 H)$ had a value of $u_{*}=0.305 \mathrm{~m} \mathrm{~s}^{-1}$. This results in relations of $u_{*} / U_{2 H}=$ 0.131 and $u_{*} / U_{e}=0.0828$, where $U_{2 H}=2.34 \mathrm{~m} \mathrm{~s}^{-1}$ is the horizontal velocity magnitude at twice the building height and $U_{e}=3.69 \mathrm{~m} \mathrm{~s}^{-1}$ the free-stream velocity at $z / H=12$. The roughness length $z_{0} / H=0.076$ was determined from a fit of the logarithmic wind profile with a von Kármán constant of $\kappa=0.39$ and using a zero-plane displacement height $d / H=0.58$ that was computed before from the pressure and shear stress distributions on the walls using Jackson's [48] approach (see Castro et al. [23] for details). For the purpose of non-dimensionalising the results from the LES, we use $H=1 \mathrm{~m}$.

\section{Dispersion models}

The dispersion modelling approaches and set-ups of the specific models used in this study are described below. A summary is presented in Tab. 2. In all formulations below and in Sect. 4 and 5, a Cartesian coordinate system is used that is aligned with the streets of the DIPLOS array (see Fig. 2c), where $x, y, z$ denote lateral and vertical directions. Time-averaged variables are written in upper case letters, i.e. $c=C+c^{\prime}$, where $C=\bar{c}$ is the time mean, $c^{\prime}$ the fluctuation about the mean and $c$ the instantaneous value. Volume-averaged quantities are indicated by square brackets, $[C]$; spatial averages over 2D facets/areas by angled brackets, $\langle C\rangle$. 
3.1 Gaussian plume model

We use the Gaussian plume model formulation introduced by Hanna et al. [42] as a baseline urban dispersion model. Previous evaluations of this model against two field experiments showed a satisfactory performance in high-density, high-rise urban environments, for which a priori information on the initial lateral and vertical plume spread were provided to the model [39]. While there are certainly more sophisticated (operational) Gaussian dispersion models available (see Sect. 1.2), they share the same underlying modelling framework with Hanna et al.'s baseline model, which will therefore be the subject of interest in our model inter-comparison.

In the model formulation used on this study, the spatial distribution of the mean scalar concentration $C$ originating from a continuous point-source release is given by the classic Gaussian plume equation with ground reflection at $z=0 \mathrm{~m}$

$$
\begin{aligned}
C(x, y, z)= & \frac{Q}{2 \pi U_{p} \sigma_{y} \sigma_{z}} \exp \left(-\frac{y^{2}}{2 \sigma_{y}^{2}}\right) \\
& \times\left[\exp \left(-\frac{\left(z-h_{Q}\right)^{2}}{2 \sigma_{z}^{2}}\right)+\exp \left(-\frac{\left(z+h_{Q}\right)^{2}}{2 \sigma_{z}^{2}}\right)\right],
\end{aligned}
$$

where $U_{p}$ is a representative UCL wind speed, $Q$ is the constant mass emission rate and $h_{Q}$ the release height ( $h_{Q}=0 \mathrm{~m}$ in this study). The dispersion coefficients, $\sigma_{y}$ and $\sigma_{z}$, are given by the classic Briggs [12] parametrisations for urban areas, including a modification of the lateral plume spread parameter, $\sigma_{y}$, for light-wind situations proposed by Hanna et al. [42]:

$$
\begin{gathered}
\sigma_{y}=\sigma_{y_{0}}+\max \left(0.16,\left(A / U_{p}\right)\right) x /(1.0+0.0004 x)^{-\frac{1}{2}}, \\
\sigma_{z}=\sigma_{z_{0}}+0.14 x /(1.0+0.0003 x)^{-\frac{1}{2}}
\end{gathered}
$$

where $A=0.25 \mathrm{~m} \mathrm{~s}^{-1}$. Hence, the modification in Eq. (2) comes into play when $U_{p}$ is less than about $1.6 \mathrm{~m} \mathrm{~s}^{-1}$. The initial plume spread is set to $\sigma_{y_{0}}=\sigma_{z_{0}}=H / 3$, which is lower than the value of $H / 2$ proposed by Hanna et al., but leads to more realistic results in terms of the initial upwind spread for the scenario investigated here, where the source is located in a street with strong flow channelling. It has to be noted that such a priori knowledge about the flow in the source street is usually not available when running dispersion models for emergency-response scenarios. As in the LES the spatial resolution was uniform in all direction with a grid spacing of $\Delta=H / 16$.

The bulk travel speed of the plume within the canopy layer, $U_{p}$, was approximated by spatially averaging the horizontal flow from the LES over a depth of $z=0 \mathrm{~m}$ to $H$, resulting in a canopy-layer advection velocity of $U_{c}=0.67 \mathrm{~m} \mathrm{~s}^{-1}$. In actual operational dispersion modelling the cloud speed cannot usually be derived from such detailed, space-resolved information as was the case here. Instead, this quantity has to be approximated through parametrisations based on more accessible quantities. We note here that the value of $U_{c}$ stated above is quite close to the value of $0.73 \mathrm{~m} \mathrm{~s}^{-1}$ determined from the relationship $U_{c}=u_{*}\left(2 / \lambda_{f}\right)^{1 / 2}$ proposed by Bentham and Britter [7] where $u_{*}$ is the LES friction velocity. Hanna and Britter [43] suggest the relation $U_{c}=0.45 U_{2 H}$ for typical built-up inner-city areas with $\lambda_{f}>0.3$ (as in our study), which results in a value of $1.05 \mathrm{~m} \mathrm{~s}^{-1}$ based on $U_{2 H}=2.34 \mathrm{~m} \mathrm{~s}^{-1}$ in the LES (Sect. 2.2.2). 
Table 2: Overview of dispersion model set-ups used in this study.

\begin{tabular}{|c|c|c|}
\hline Name & Type & Flow \\
\hline GAUSS-1 & Gaussian plume & $\begin{array}{l}\text { LES mean UCL velocity; } \\
\text { RSL plume deflection }\end{array}$ \\
\hline GAUSS-2 & Gaussian plume & $\begin{array}{l}\text { LES mean UCL velocity; } \\
\text { UCL plume deflection }\end{array}$ \\
\hline QUIC (URB) & Lagrangian stochastic & $\begin{array}{l}\text { QUIC-URB } \\
\text { (diagnostic model) }\end{array}$ \\
\hline QUIC (CFD) & Lagrangian stochastic & $\begin{array}{l}\text { QUIC-CFD } \\
\text { (prognostic model) }\end{array}$ \\
\hline QUIC (LES) & Lagrangian stochastic & $\begin{array}{l}\text { 3D LES field } \\
\text { (prognostic model) }\end{array}$ \\
\hline UoR-SNM & street network & LES velocities \\
\hline SIRANE-1 & street network & parametrisations \\
\hline SIRANE-2 & street network & LES velocities \\
\hline
\end{tabular}

To add some degree of building-awareness, the average horizontal plume deflection was taken into account. Two deflections from the $-45^{\circ}$ forcing direction were considered: (1) based on the average horizontal wind direction of $-54^{\circ}$ determined from the LES over a depth of $1 \leq z / H \leq 2$, covering the roughness sublayer (RSL) and (2) based on the LES UCL-averaged horizontal wind direction of $-78^{\circ}$. While the former is a quantity that could be approximated through measurements in an emergency, e.g. from tower or roof-level measurements, the latter is usually not easily obtainable from sparse in-situ measurements within the canopy layer. Initial tests of the model have shown a high sensitivity of the results to the plume-turning parameter in comparison to the plume orientation observed on the LES and the wind tunnel.

\subsection{Lagrangian dispersion model}

We use the Quick Urban \& Industrial Complex (QUIC) dispersion modelling system (v6.2 and v6.29) developed by LANL and the University of Utah [53]. The core of the system is the Lagrangian model QUIC-PLUME that introduces additional terms to the classic Langevin random-walk equations in order to account for urban dispersive effects arising from spatial inhomogeneities of UCL turbulence and particle reflections on surfaces. A detailed description of the model components is presented by Williams et al. [81].

In this study, QUIC-PLUME is run on 3D wind fields from two system-integrated flow models. (i) the building-aware mass-consistent wind solver QUIC-URB that is based on the empirical-diagnostic modelling strategy developed by Roeckle [59] and expanded upon by Brown et al. [17]. QUIC-URB computes mean wind fields around buildings by using empirical relationships to produce backflow in low pressure zones (e.g. street canyons) in combination with a mass consistency constraint which results in flow recirculation in the regions of interest [55]. (ii) QUIC-CFD that is based on the RANS equations in combination with a zeroth-order turbulence model using a mixing-length approach [37]. In order to disentangle the performance of the dispersion model from the accuracy of the wind models, in the final variant (3), QUIC-PLUME is driven directly by the mean 3D LES reference wind field.

In both QUIC-URB and QUIC-CFD a logarithmic wind profile for neutral stratification is prescribed at the inflow edges based on the LES roughness parameters and $H$ was set to 
$16 \mathrm{~m}$. The reference wind speed $U_{\text {ref }}$ was $4 \mathrm{~m} \mathrm{~s}^{-1}$ in a height of $z_{\text {ref }}=4.5 H$. In agreement with the LES, a uniform grid resolution of $\Delta=H / 16$ was used. In order to ensure a fully converged wind environment upstream of the source, the DIPLOS array set-up was realised in a slightly larger domain of $28 H \times 27 H \times 12 H$. QUIC-URB was run with the recommended settings [53], including a modified wake-zone model [52]. For QUIC-CFD, model parameters like the time step or the maximum allowable mixing length were automatically generated by the system based on the specified geometry, cell size and wind speed.

With $\Delta_{x}=\Delta_{y}=H / 8$ and $\Delta_{z}=H / 16$ the collecting boxes for the particles were slightly larger than the flow grid cells in order to reduce the statistical noise of the output. As in the LES, computational particles with passive-tracer characteristics were released continuously through a circular area ground source with a diameter of $0.244 H$. In each run, 612,000 particles were released over a duration of $30 \mathrm{~min}$. The model time step was set to $0.1 \mathrm{~s}$.

\subsection{Street-network dispersion models}

The street-network dispersion modelling approach is based on the balance equation for the volume-averaged scalar concentration $[C]_{V}$ within a street or intersection box of volume $V$ in the UCL

$$
\frac{d[C]_{V}}{d t}+\frac{1}{V} \sum_{k=1}^{K} \Phi^{k}=[Q]_{V}
$$

where $[Q]_{V}$ is the volume-source term and $\Phi^{k}$ is the total scalar flux through the $k$ th facet of the box $[67,38,6]$. The total scalar flux $\Phi^{k}$ can be partitioned into an advective and a turbulent component. The horizontal exchange between street and intersection boxes is assumed to be mainly advective and the associated scalar flux is

$$
\Phi_{a d v}^{k}=C U_{i} A^{k} \equiv[C]_{V} U_{i}^{k} A^{k}
$$

where $U_{i}^{k}(i=1,2)$ is the horizontal advection velocity aligned with the street, with which material is transported through facet $k$ of area $A^{k}$. The inherent assumption of this approach is that the material is well-mixed within each street or intersection box, i.e. spatial concentration fluctuations are small compared to the spatial mean.

On the other hand, the vertical exchange between the UCL and the external flow above the buildings is assumed to be mainly turbulent and can be approximated by an exchange velocity approach

$$
\Phi_{\text {turb }}^{\text {top }}=\overline{c^{\prime} w^{\prime}} A^{\text {top }} \equiv\left([C]_{V}-[C]_{e x t}\right) E A^{t o p},
$$

where $E$ is the vertical turbulent exchange velocity through the top facet of the box that has an area of $A^{t o p}$ [71]. The direction of exchange is determined by the difference between the UCL and external concentrations, $[C]_{V}-[C]_{e x t}$. Figure 3a schematically illustrates the flux balance for a street box. Dispersion above the canopy, where the street-network concept breaks down, has to be modelled by a different approach, e.g. using a Gaussian plume model. 


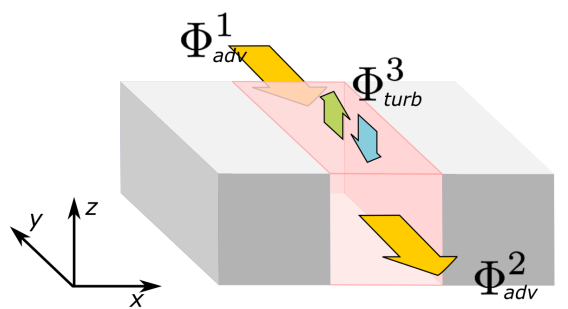

(a)

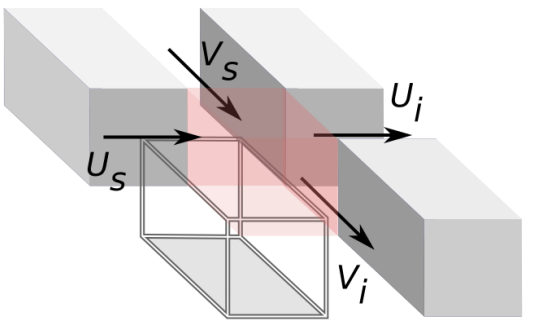

(b)

Fig. 3: (a) Flux balance for a street box: Material is transported into the box through facet $1\left(\Phi_{a d v}^{1}\right)$ and out of the box through facet $2\left(\Phi_{a d v}^{2}\right)$. Through the top facet, the box can gain or lose material through turbulent exchange with the external field $\left(\Phi_{\text {turb }}^{3}\right)$. (b) Horizontal advection velocities defined in the UoR street-network model (UoR-SNM). The index 's' denotes flow coming out of a street, the index ' $\mathrm{i}$ ' is for flow coming out of an intersection.

\subsubsection{UoR-SNM}

For a general demonstration of the street-network modelling approach, we use the University of Reading Street-Network Model (UoR-SNM) introduced by [6], where a detailed derivation of the model formulation is presented. This model was previously tested against DNS dispersion data in a cube-array environment $[35,36]$.

In contrast to the fully operational street-network model SIRANE, UoR-SNM does not include built-in flow parametrisations. Instead, we use a hybrid approach by deriving from an external flow simulation the velocity parameters, $U_{i}^{k}$ and $E$, required by the model to compute the horizontal and vertical concentration fluxes based on Eqs. (5) and (6). In this study, we use the LES data for this purpose. Hence, the main aim of including UoR-SNM in the inter-comparison study is to demonstrate the viability of the approach and to highlight its strengths and limitations with regard to the representation of dispersion processes.

Horizontal advection velocities, $U_{i}^{k}$, used in Eq. (5) were obtained from the LES in terms of facet-averages of the time-mean velocity components along $x$ and $y$ streets as shown in Fig. 3b. In order to account for upwind transport of scalars, a diffusive transport component was added in the UCL together with an additional transport term into sheltered regions (here into $x$ streets) following Hamlyn et al. [38]. The vertical turbulent exchange velocity used in Eq. (6) at the top of the canopy layer $(z / H=1)$ was derived from the LES according to

$$
E=\frac{\left\langle\overline{c^{\prime} w^{\prime}}\right\rangle_{z / H=1}}{[C]_{u c l}-[C]_{e x t}}
$$

where $[C]_{u c l}$ are LES concentrations averaged over street and intersection volumes within the UCL $(0 \leq z / H \leq 1)$ and $[C]_{\text {ext }}$ box-averaged concentrations in the external flow over a depth of $1 \leq z / H \leq 2$ [5]. Exchange velocities were computed for $x$ streets $\left(E_{x}\right), y$ streets $\left(E_{y}\right)$ and intersections $\left(E_{i}\right)$ individually. Dispersion above the canopy was modelled in UoRSNM by a simple advection-diffusion approach using the same box discretisation as in the UCL and mean horizontal transport velocities derived from the LES. 


$$
E=\frac{\sigma_{w}}{\sqrt{2} \pi}
$$

and $\sigma_{w}$ is parametrised for different stability ranges via $u_{*}$. Another difference between UoR-SNM and SIRANE is the treatment of dispersion through intersections. In the former, the intersection is assumed to be well-mixed and fluxes out of the intersection into downwind streets are parametrised through the advection velocities $U_{i}$ and $V_{i}$ (Fig. 3b). In SIRANE, mixing in the intersection and the $2 \mathrm{D}$ branching of material is determined by the external flow using a model for the volume-flux conservation. This approach takes into account the local geometry, the external wind direction and the standard deviation of its fluctuations [68, 71]. Imbalances are overcome by vertical exchange with the external flow.

We ran SIRANE in two modes: (1) with the default flow parametrisations described above; (2) with the LES flow information provided as in the case of UoR-SNM. Since SIRANE treats intersections purely as nodal points connecting adjacent streets, only the vertical exchange velocities in the $x$ and $y$ streets need to be parametrised together with the horizontal advection velocities along each street, $U_{s}$ and $V_{s}$. In order to adjust the external flow field to the reference conditions, in both cases the average horizontal wind direction in the LES over $1 \leq z / H \leq 2\left(-54^{\circ}\right)$ was prescribed as the forcing direction.

\section{Flow and dispersion characteristics}

In the following all flow and concentration quantities are presented in a non-dimensional framework. Non-dimensional concentrations, $C^{*}$, and concentration fluxes, $\overline{c^{\prime} u_{i}^{\prime}}$, are computed as

$$
C^{*}=\frac{C U_{r e f} H^{2}}{Q}
$$

and

$$
\overline{c^{\prime} u_{i}^{\prime}}=\frac{\overline{c^{\prime} u_{i}^{\prime}} H^{2}}{Q},
$$

where $Q$ is the constant mass emission rate and $U_{r e f}$ is the mean streamwise reference velocity defined in the approach-flow coordinate system in a height of $4.5 \mathrm{H}$. 


\subsection{Flow behaviour}

The quality of dispersion predictions to a large degree depends on whether the underlying flow as the main physical driver of advection and mixing processes is adequately represented. Both SIRANE and the QUIC modelling suite are complete operational systems that include means of calculating all necessary flow information in the UCL and the external boundary layer that is required by the dispersion modules. In order to understand the concentration output it is therefore crucial to also appraise the adequacy of the flow modelling.

QUIC-PLUME requires the most detailed flow information in terms of a full 3D representation of the mean flow. Previously, [54] evaluated the performance of QUIC-URB and QUIC-CFD against wind data measured during the Joint Urban 2003 field campaign in Oklahoma City and found that both wind models performed similarly well. When tested in an idealised cube-array geometry, which is closer to the DIPLOS set-up regarding the degree of geometrical abstraction, [65] found that building-induced flow features in QUIC-URB compared well with wind-tunnel data.

Figure 4 shows wind vectors and vertical mean velocities of the LES and the two QUIC wind models for the DIPLOS case. For $-45^{\circ}$ and other model orientations, Castro et al. [23] previously validated the LES flow against the wind-tunnel measurements and found that the salient features of the complex UCL flow patterns agree as well as can be expected with the experiment given the uncertainties described in Sect. 2.2.1.

The data is shown in a horizontal plane at $z / H=0.5$ in terms of an ensemble-average over the time-averaged flow in all repeating units of the domain (Fig. 2b). Doubling the length of one building side introduced a geometrical asymmetry for which the resulting flow patterns deviate strongly from the corresponding cube-array case with its symmetric corner vortices and flow convergence in intersections (e.g. Fig. 4 in Coceal et al. [26]).

The LES shows a fully developed channelling region along the $y$ street through the intersection, cutting off most of the outflow from the $x$ street, where a strong recirculation pattern is established. This is also reflected in the histogram of LES mean horizontal wind directions, $\theta$, over the entire UCL (Fig. 5), which reveals a strong peak at $-90^{\circ}$ (flow in $-y$ direction) and only a small plateau between $0^{\circ}$ and $90^{\circ}$. The intersection shows a highly three-dimensional flow structure. Alternating regions of up-drafts and down-drafts in both streets indicate recirculation patterns in the vertical plane. In combination with the observed along-street channelling this results in a helical recirculation [29] along the $y$-street canyon, which extends well into the intersection. Channelling in the long streets was also observed in the MUST geometry at a similar inflow angle [28], but the larger street width resulted in weaker flow deflection and also in less well-established flow recirculation in the short street. Unlike the LES, the histograms of both QUIC wind models show peaks at the forcing direction of $-45^{\circ}$ (Fig. 5). In the case of QUIC-CFD, this is mainly due to the flow behaviour in the intersections and the flow entering the long streets, which has a stronger $u$-component compared to the LES. The general patterns of updraft regions protruding from the leeward building sides well into the intersection and downdraft regions on the windward sides are very similar in the LES and QUIC-CFD. In contrast to that, in QUIC-URB the helical flow does not extend into the intersection but is confined to the long street. In both QUIC flow models, the recirculation zone in the short street is much larger and less confined compared to the LES. Here, QUIC-URB shows a strong flow reversal into $-x$ direction (peak at $\pm 180^{\circ}$ in Fig. 5) and also predicts a stronger negative $u$-component in the $y$-street compared to the two prognostic flow simulations (peak at about $-110^{\circ}$ ). Whereas in the LES and QUIC-CFD, the flow pattern in the intersection is determined by the channelling in the long street, in QUIC-URB the outflow from the long streets is entering the 


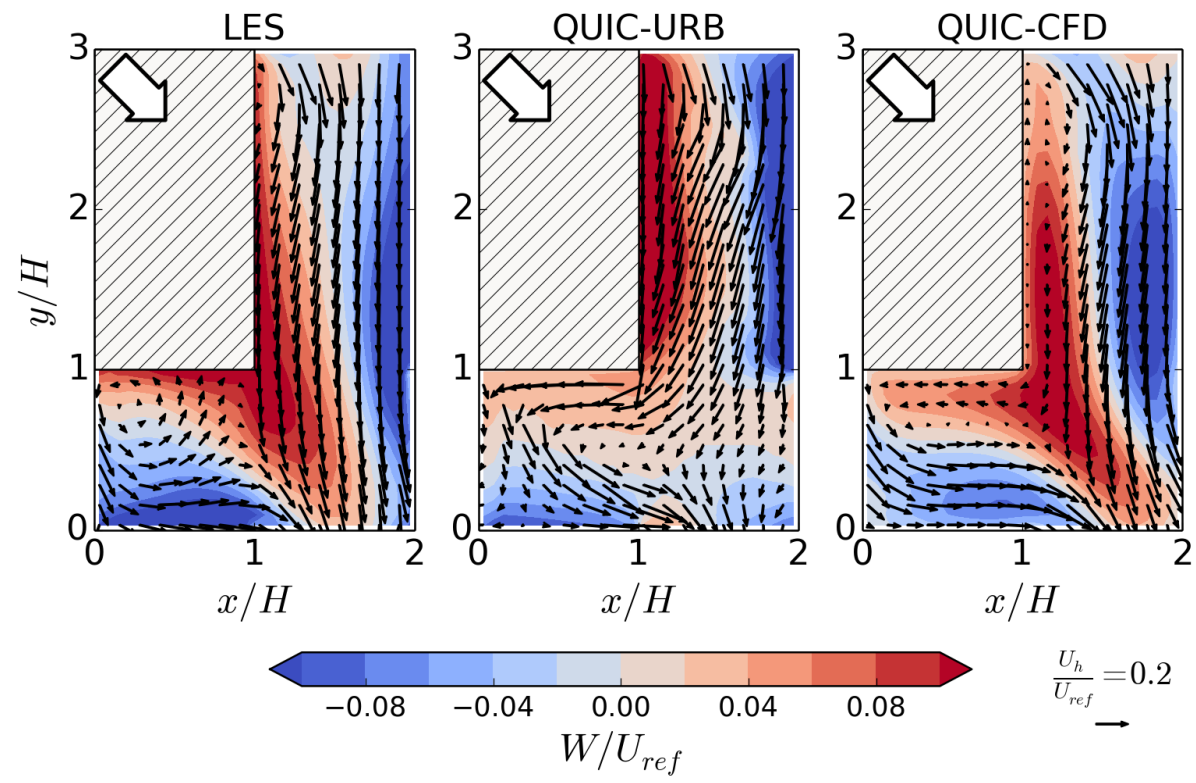

Fig. 4: Horizontal cross-sections at $z / H=0.5$ showing mean horizontal velocity vectors and vertical velocities of the LES (left), QUIC-URB (centre) and QUIC-CFD (right). The data represent ensemble averages over all repeating units of the array (see Fig. 2b). The length of the vectors scales with the mean wind speed $U_{h}=\sqrt{U^{2}+V^{2}}$. Note that only every fourth vector is shown. Large arrows indicate the forcing wind direction.

upwind short street and the intersection flow largely reflects the recirculating flow pattern. These differences are expected to have an influence on the topological dispersion behaviour through the street network.

\subsubsection{Horizontal advection velocities}

Mean horizontal advection velocities as defined in Fig. 3b were computed from the LES in terms of facet-averaged mean velocities at the four interfaces between street and intersection boxes. This resulted in values of $\left\langle U_{i}\right\rangle=0.22 \mathrm{~m} \mathrm{~s}^{-1}$ and $\left\langle V_{i}\right\rangle=-0.71 \mathrm{~m} \mathrm{~s}^{-1}$ for flow out of the intersection into the downwind $x$ and $y$ streets, respectively, and $\left\langle U_{s}\right\rangle=0.23 \mathrm{~m} \mathrm{~s}^{-1}$ and $\left\langle V_{s}\right\rangle=-0.77 \mathrm{~m} \mathrm{~s}^{-1}$ for flow from the streets into the intersections (for $U_{\text {ref }}$ of $3 \mathrm{~m} \mathrm{~s}^{-1}$ at $z_{\text {ref }}=4.5 H$ ). As a result of the flow channelling along the $y$ streets (Fig. 4), the magnitudes of advection velocities along the $y$-axis, $\langle V\rangle$, exceed those along the $x$-axis, $\langle U\rangle$, by more than a factor of 3. Similar ratios are observed in the experiment, with the important caveat that here we compare point values measured at the interfaces in heights of $z / H=0.5$ and not averages over the entire facets from the ground to roof level. Based on the same reference velocity as in the LES, from the wind-tunnel flow measurements we obtain: $U_{i}=0.21 \mathrm{~m} \mathrm{~s}^{-1}$, $V_{i}=-0.51 \mathrm{~m} \mathrm{~s}^{-1}, U_{s}=0.18 \mathrm{~m} \mathrm{~s}^{-1}$ and $V_{s}=-0.58 \mathrm{~m} \mathrm{~s}^{-1}$. Note that here we used flow data measured on a much denser grid in a small region of the array compared to the relatively coarse mapping grid shown in Fig. 2c. 


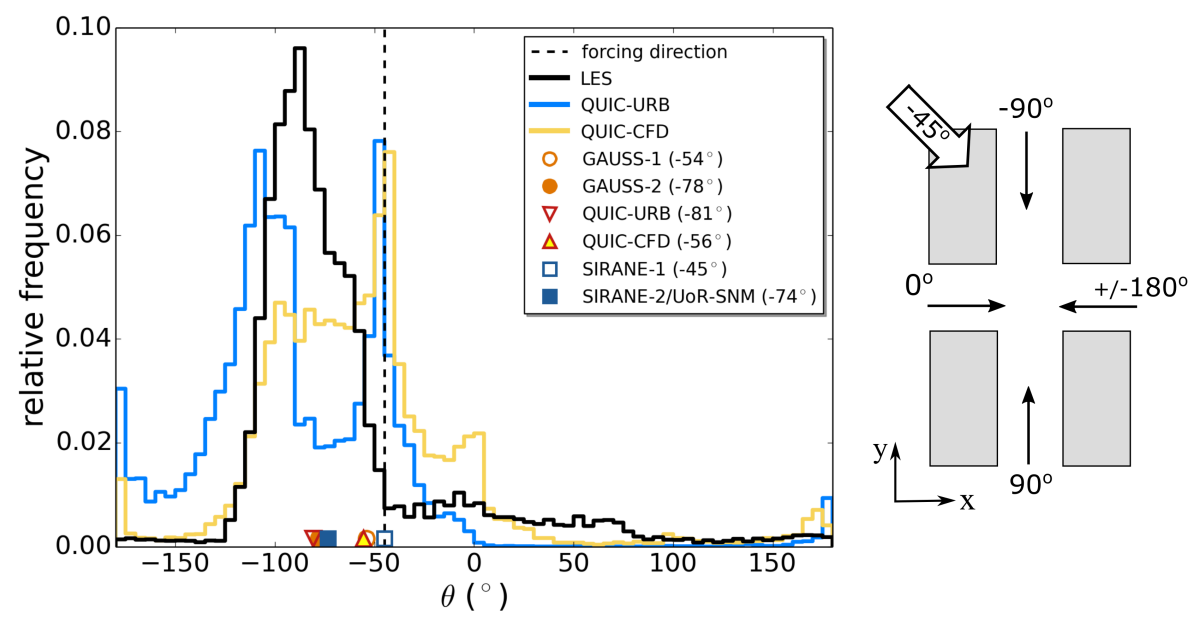

Fig. 5: Histograms of horizontal wind directions, $\theta$, in the UCL $(0 \leq z / H \leq 1)$ derived from LES, QUIC-URB and QUIC-CFD mean flow fields at a forcing wind direction of $-45^{\circ}$. A wind direction of $-90^{\circ}$ represents flow into negative $y$ direction; $0^{\circ}$ flow into positive $x$ direction as indicated in the schematic on the right. The plume directions for the Gaussian model runs are shown together with the canopy-layer plume direction derived from the bulk horizontal advection velocities in the Lagrangian and street-network models (see Tab. 3).

Table 3 contrasts these results with the advection velocities modelled in SIRANE-1 and the equivalents from both QUIC wind models. Unlike the LES, the parametrisation in SIRANE-1 produces the same velocity magnitudes for $\left\langle U_{s}\right\rangle$ and $\left\langle V_{s}\right\rangle$. As a result, the dispersion module will be unaware of the strong change in flow direction within the canopy layer and resulting pollutant channelling effects. The transport velocity along the short $x$ street is significantly over-predicted compared to the LES, yet a much better agreement is found for $\left\langle V_{s}\right\rangle$ along the longer $y$ streets. Currently the SIRANE velocity model formulation does not take into account effects of the street length, but instead assumes a fully developed flow as through an 'infinite' street. The shorter the street, the less applicable this assumption becomes. However, a mere factor-of-2 increase of the $y$-street lengths compared to the $x$ streets in the DIPLOS array already resulted in a good agreement with the LES. The advection velocities derived from the QUIC wind models support the previous assessments. In QUIC-CFD the channelling in $y$ direction and through large parts of the intersections resulted in an exceedance of magnitudes of $\langle V\rangle$ compared to $\langle U\rangle$ by about a factor of 1.4, which is less than half of the factor in the LES and also much lower compared to the experiment. For QUIC-URB, on the other hand, there is more than a factor of 6 difference between the outflow from the long and the short streets, i.e. twice the factor seen in the LES. Here, the low value of $\left\langle U_{s}\right\rangle$ results from the flow reversal along the facet triggered by the recirculation regime; $\left\langle U_{i}\right\rangle$ is similarly small as there is less outflow from the intersection into the downwind short street compared to the LES and QUIC-CFD. The different flow orientations based on the advection velocities listed in Tab. 3 are summarised in Fig. 5 together with the prescribed values for the two Gaussian model runs. 
Table 3: Horizontal advection velocities and vertical turbulent exchange velocities derived from the LES together with modelled parameters from SIRANE-1. Velocity parameters derived from the LES are used in UoR-SNM and SIRANE-2. Corresponding advection velocities from both QUIC wind models are included for comparison. All velocities have units of $\mathrm{m} \mathrm{s}^{-1}$ and correspond to a reference velocity $U_{\text {ref }}$ of $3 \mathrm{~m} \mathrm{~s}^{-1}$ at $z_{\text {ref }}=4.5 \mathrm{H}$.

\begin{tabular}{lccccccc}
\hline Model & $\left\langle U_{s}\right\rangle$ & $\left\langle V_{s}\right\rangle$ & $\left\langle U_{i}\right\rangle$ & $\left\langle V_{i}\right\rangle$ & $E_{x}$ & $E_{y}$ & $E_{i}$ \\
\hline LES & 0.23 & -0.77 & 0.22 & -0.71 & 0.10 & 0.15 & 0.12 \\
SIRANE-1 & 0.84 & -0.84 & - & - & 0.09 & 0.09 & - \\
QUIC-URB & 0.10 & -0.63 & 0.12 & -0.68 & - & - & - \\
QUIC-CFD & 0.42 & -0.61 & 0.40 & -0.55 & - & - & - \\
\hline
\end{tabular}

\subsection{Dispersion behaviour}

Before discussing the results of the model inter-comparison study in Sect. 5, in the following paragraphs some general features of the dispersion scenario are presented based on the LES and wind-tunnel data.

\subsubsection{Plume characteristics}

Figure 6 shows the 3D LES plume in terms of a concentration iso-surface at $C^{*}=0.01$. The overall plume shape is strongly non-Gaussian and the material is distributed asymmetrically about the forcing wind direction of $-45^{\circ}$. Vertically the plume extends up to approximately $z / H=5$ in the region covered by the simulation. The plume shape implies that within the building array, material is transported along the $y$ direction downwind of the source, where there is significant detrainment of material out of the UCL. Above the array pollutant pathways adjust to the forcing wind direction. Differences in concentration distributions within and above the UCL are further illustrated in Fig. 7, showing LES mean concentrations in the $(x, y)$ plane together with corresponding point-wise wind-tunnel measurements at $z / H=0.5$ and 1.5. The agreement between LES and experiment regarding the shape of the plume footprints and the local concentration levels is satisfactory. The extent of the plumes in $+x$ direction agrees very well, also with regard to the level of upwind spread of material from the source street. Both LES and experiment show strong channelling of the plume down the source street, which overall leads to an asymmetric plume footprint. Some differences in the plume shapes and concentration levels farther away from the source can be determined. Some of these could be attributable to positional uncertainties of the wind-tunnel data in any horizontal plane as discussed in Sect. 2.2.1, which can be as large as $0.06 \mathrm{H}$ in the vertical. However, there seems to be a slight systematic difference in the orientation of the plumes in the UCL, which becomes more effective further downwind of the source. Here we note that the lowest two rows of measurements (around $y / H=-15$ ) were taken only one block away from the edge of the model (see Fig. 2a) and there is an increase in uncertainties attached to the concentration measured far downwind of the source. For further discussions of the general comparison between experiment and LES see Fuka et al. [32].

The LES plume centreline in the canopy, here defined as the line of maximum concentration downwind of the source, proceeds along the $y$ axis $(x / H=0)$ and thus is offset by $45^{\circ}$ to the external flow as a result of the flow channelling in the long streets. The near-field behaviour of the plume is similar to the MUST case reported by Dejoan et al. [28] for a similar scenario. However, due to the narrower streets in the DIPLOS array, channelling effects 


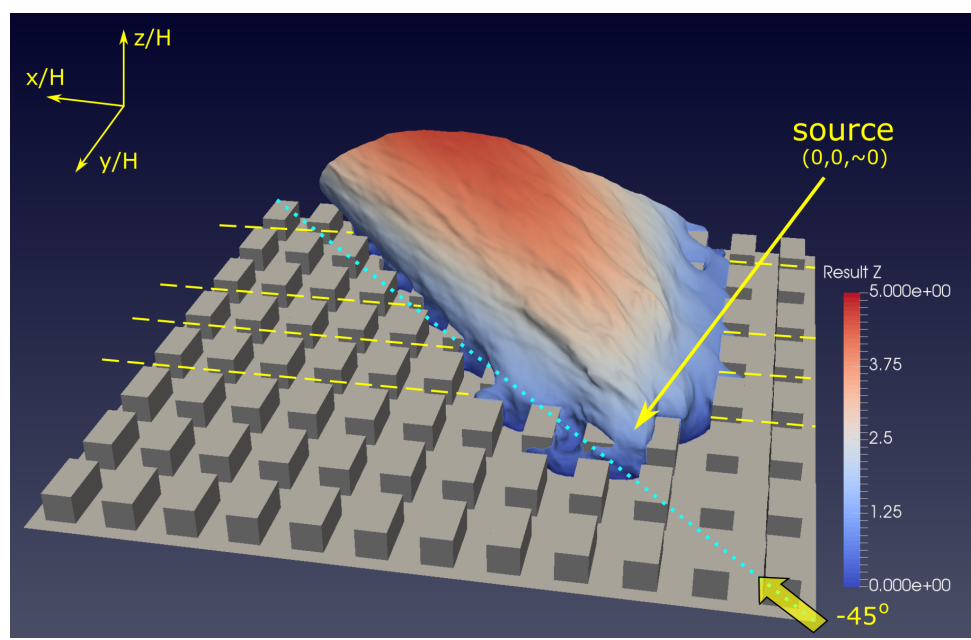

Fig. 6: $C^{*}=0.01$ iso-surface of the LES plume looking into downwind direction. Colour contours on the plume indicate the height above ground, $z / H$. The position of the ground source and the forcing wind direction are indicated (large arrow; dotted line along $-45^{\circ}$ ). Dashed lines show the locations of the $(x, z)$ cross sections discussed in Fig. 8.
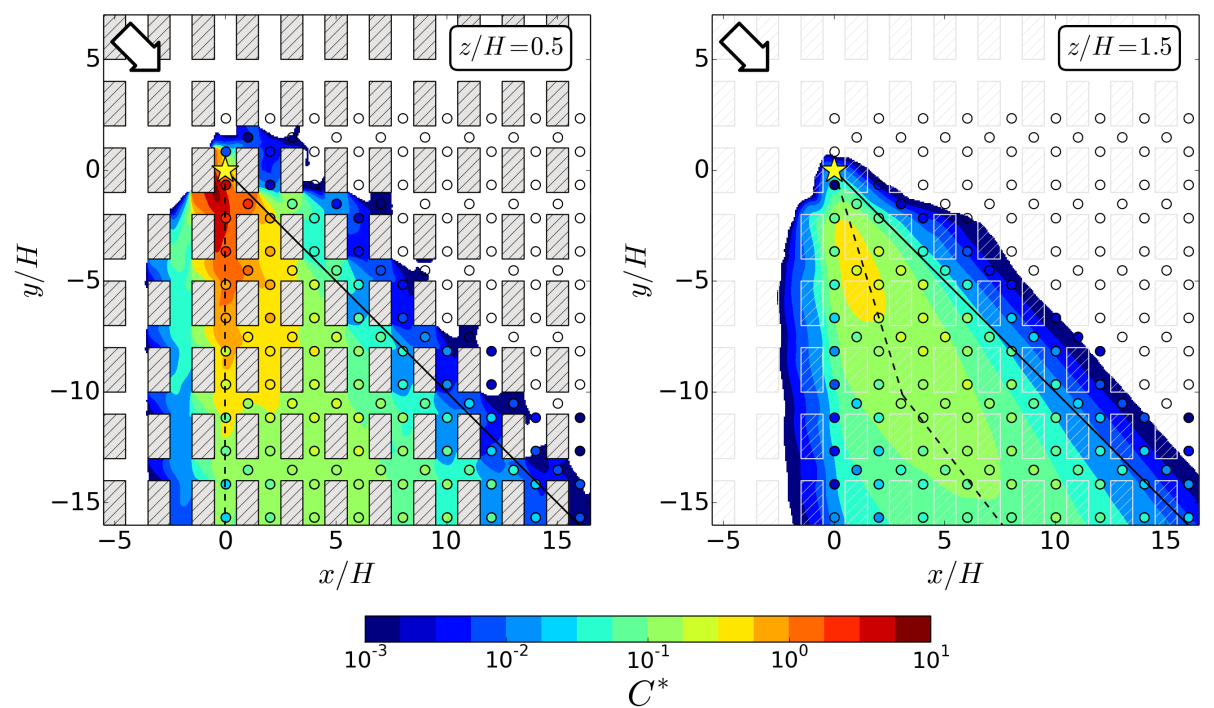

Fig. 7: Mean concentrations in the horizontal plane at $z / H=0.5$ (left) and $z / H=1.5$ (right). Contours represent the LES data and circles the point-wise wind-tunnel measurements. Empty circles for the wind tunnel show measurement sites where the $C^{*}$ was less than $10^{-3}$ and the experimental data can be subject to large uncertainties. Solid black lines show the forcing wind direction; dashed black lines indicate the approximated LES plume direction based on the maximum mean concentration in the horizontal plane. In this and all following figures, arrows indicate the forcing direction and stars the source location. 
are much stronger and comparable to observations in other idealised street networks [33] or realistic urban centres [82]. As the flow above the canopy re-adjusts back to the forcing direction, the offset of the plume centreline decreases. This is particularly the case in the downwind regions of the plume, where less material is detrained from the canopy (Fig. 7). A similar shift can also be seen in the wind-tunnel data.

\subsubsection{Vertical exchange}

The vertical transport of pollutants out of and back into the canopy layer plays a defining role in the dispersion scenario investigated here. Concentration distributions and turbulent exchange characteristics in the vertical $(x, z)$ plane are shown in Fig. 8 for four fixed $y / H$ positions downstream of the source as indicated in Fig. 6. While in the UCL the concentration maxima are located at $x / H=0$ over the entire $y$ extent of the plume, above the buildings the plume is advected into $+x$ direction with the re-adjusting flow. Due to the higher velocities here, the material is transported much faster horizontally than in the canopy layer. Already at a distance from the source of $4.5 \mathrm{H}$ in $-y$ direction, a significant part of the plume is located outside of the UCL. The corresponding fields of the vertical turbulent momentum flux, $\overline{c^{\prime} w^{\prime}}$ * , show that the detrainment of material out of the UCL is strongest close to the source as seen in the slices at $y / H=-1.5$ and -4.5 , while in the far-field of the plume the exchange is directed back into the canopy and is strongest in the shear layer just above rooflevel $(y / H=-13.5)$. The cross section at $y / H=-7.5$ indicates an intermediate regime. This agrees with previous findings by Carpentieri et al. [19] and Goulart et al. [36].

Following Eq. (7) the vertical exchange velocity is defined at the top of each networkmodel box in the UCL. Figure 9a shows a map of $\left\langle\overline{c^{\prime} w^{\prime}}\right\rangle_{z / H=1}$ as derived from the LES by facet-averaging the high-resolution concentration flux output at the top of each street and intersection box. In the horizontal plane, distinct regions of detrainment and re-entrainment are evident. In the near-field of the source and along the plume centreline at $x / H=0$ on average the vertical turbulent concentration flux is directed out of the canopy layer at roof-level $\left(\left\langle\overline{w^{\prime} c^{\prime}}\right\rangle_{z / H=1}>0\right)$. Transport of pollutants back into the street system is dominant away from the plume centreline in lateral $+x$ direction. The regions of re-entrainment $\left(\left\langle\overline{w^{\prime} c^{\prime}}\right\rangle_{z / H=1}<0\right)$ coincide with regions where $\left[C^{*}\right]_{u c l}-\left[C^{*}\right]_{e x t}<0$ (not shown), i.e. where concentrations are higher in the external layer than in the canopy. This positive vertical concentration gradient is a result of the advection of material above the array that was detrained from streets along the plume centreline (see Figs. 6 and 8).

The spatial extent of the detrainment and re-entrainment regions respectively reflect the footprints of the main parts of the plume within and above the canopy. Figure 9a implies that surface concentrations are not exclusively governed by processes in the street network, but in certain circumstances can be controlled, locally, by the dispersion above the canopy. This is particularly important at some intermediate distance from the source, where tests with UoR-SNM for this case suggest that re-entrainment can increase streetlevel concentrations by a factor greater than 10 . In both street-network models, the vertical transfer is parametrised assuming a linear relationship between the local turbulent vertical scalar flux (facet-averaged) and the vertical concentration gradient (volume-averaged), with the exchange velocity $E$ determining the slope. The LES data for this test case supports this assumption and we find a strong positive correlation between these quantities. We also find that differences between the exchange velocities associated with upward (detrainment) and downward (re-entrainment) motions are comparable to variations in exchange efficiency for different street types. We note that the two network models used here differ in their treatment of dispersion above the canopy. In SIRANE above-roof dispersion is implemented as 

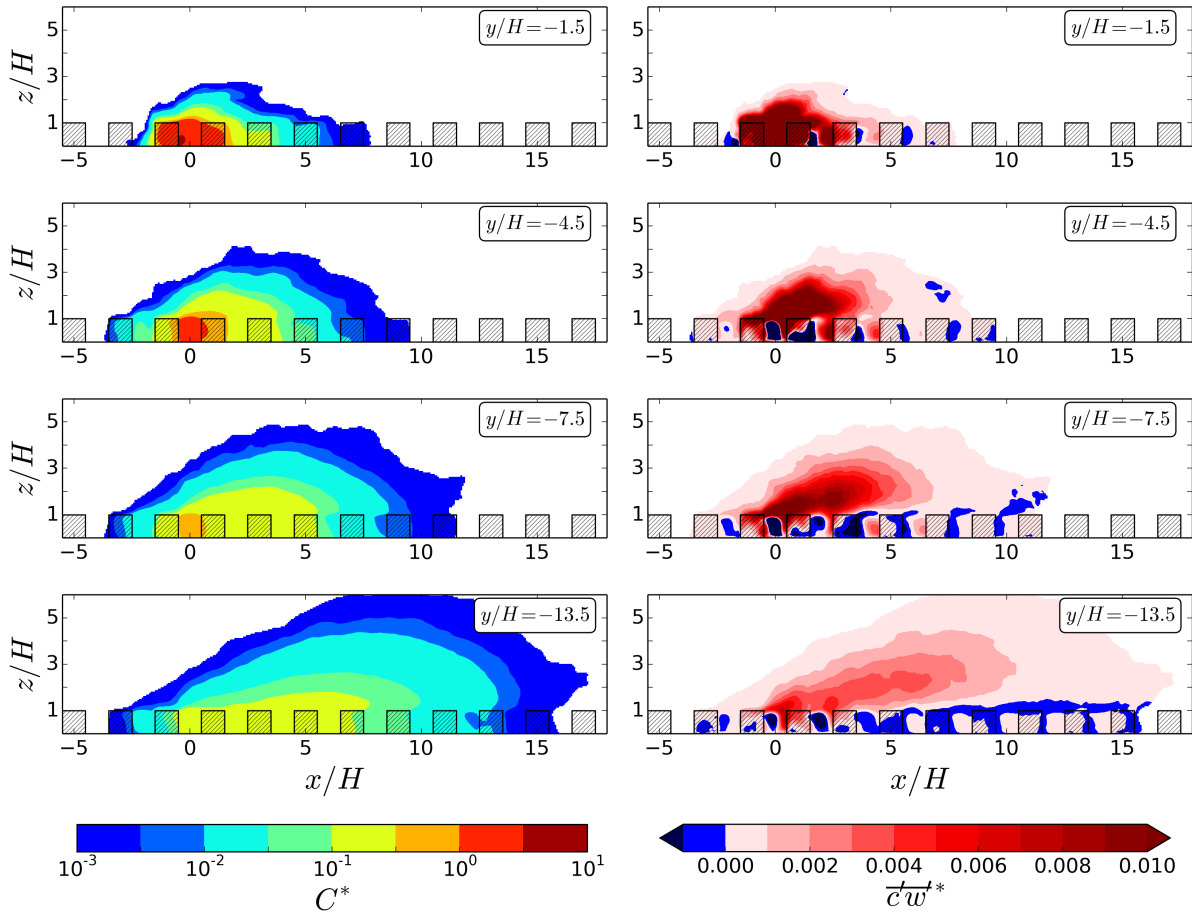

Fig. 8: Vertical $(x, z)$ cross sections of mean scalar concentrations (left) and vertical turbulent concentration fluxes (right) at four $y / H$ locations downwind of the source as indicated in Fig. 6. The source is located at $x / H=0$.

a series of point sources giving rise to Gaussian plumes that are then superimposed [71]. In UoR-SNM mean and turbulent horizontal fluxes are separately parametrised using advection velocities and diffusion coefficients in the discretised advection-diffusion equation [36].

Figure 9b compares height profiles of the LES and wind tunnel vertical concentration flux taken in a region of the plume where there is a transition between predominantly upward or downward-oriented turbulent transport (sites indicated in Fig. 9a). In both data sets, in the $x$ street (P1) and the intersection (P2) scalar fluxes are positive over all heights, while in the centre of the $y$ street (P3) the exchange around roof-level and below is negative. The quantitative agreement between LES and experiment around roof-level is very good. However, there is approximately a factor of two difference in the peak values observed at about $z / H \simeq 1$.6. Larger differences can also be observed at site $\mathrm{P} 2$ below roof-level, where the LES and experiment show opposite trends. Several reasons can explain these differences. The sites are located in a region of large spatial concentration gradients as can be seen in the $y / H=-4.5$ cross section in Fig. 8, which coincides with sites P1 and P2. The limited (but comparable) averaging times in the simulation and the experiment will cause much higher levels of uncertainty this close to the source and towards the plume edge, especially in the fluxes, where spatial concentration gradients are large and temporal signal intermittency is high compared to more well-mixed plume regions. Further uncertainties are introduced by the inevitable spatial offset between the LDA and FFID (constant downwind shift) as discussed in Sect.2.2.1. Further aspects are the slight difference in the plume orientation 


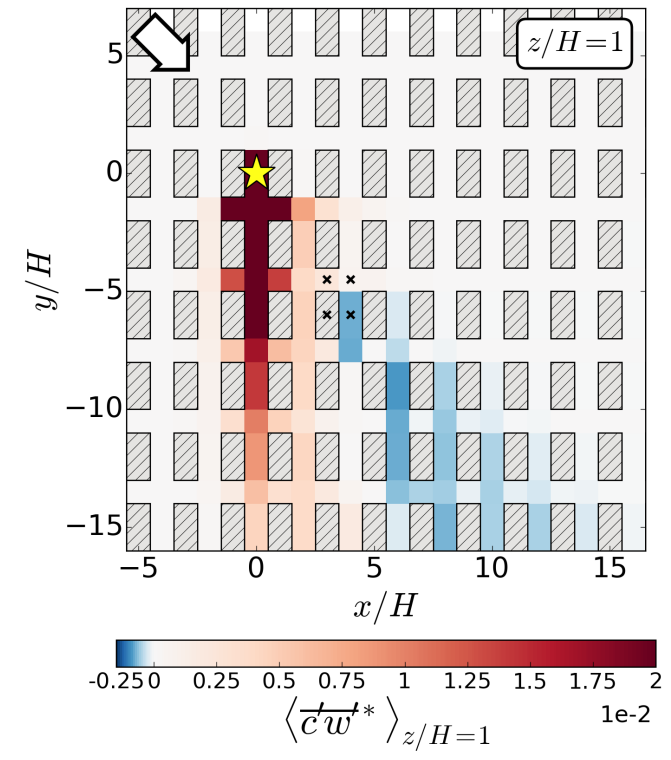

(a)
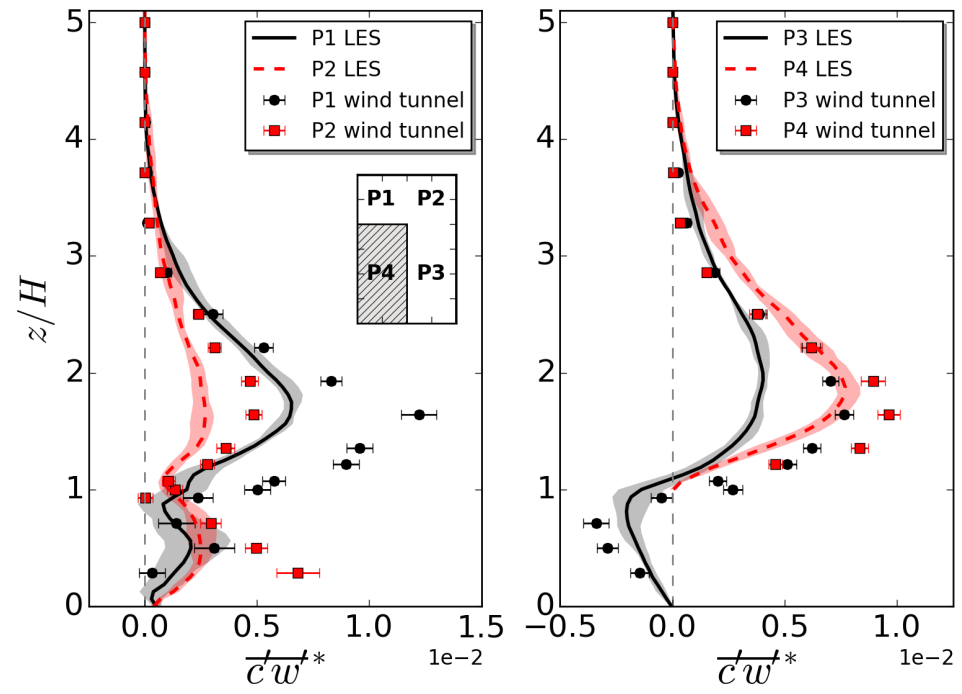

(b)

Fig. 9: (a) Facet-averaged vertical turbulent concentration flux at $z / H=1$. Crosses indicate the locations of vertical profiles measured in the wind-tunnel experiment. (b) Comparison of experimental and LES height profiles of the vertical turbulent concentration flux. Lines for the LES data represent ensemble averages and the shaded areas indicate the corresponding value range among the four ensemble members. 
in the LES and the experiment and the effects of the difference of the inflow boundary conditions in the wind tunnel (constant-direction boundary layer profile) as opposed to the fully developed periodic boundary conditions in the LES.

As discussed above, the patterns seen in Fig. 9a support the gradient-approach taken to derive the vertical exchange velocities for the UoR-SNM network model (Eq. 7). Furthermore, it was found that the turbulent component of the vertical exchange, $\left\langle\overline{c^{\prime} w^{\prime \prime}}\right\rangle_{z / H=1}$, on average is dominant compared to the advective transport, $\left\langle(C W)^{*}\right\rangle_{z / H=1}$, for this scenario in agreement with Belcher et al. [6]. Vertical exchange velocities for UoR-SNM and SIRANE2 were determined from the LES by ensemble-averaging individual results obtained in regions of significantly high flux magnitudes, resulting in $E_{x}=0.1 \mathrm{~m} \mathrm{~s}^{-1}, E_{y}=0.15 \mathrm{~m} \mathrm{~s}^{-1}$ and $E_{i}=0.12 \mathrm{~m} \mathrm{~s}^{-1}$ for $x$ streets, $y$ streets and intersections, respectively. $E_{x}$ is about $30 \%$ lower than $E_{y}$, indicating that the recirculating flow in the short street reduces the potential for vertical exchange. Applying the SIRANE parametrisation given in Eq. (8) together with the facet-averaged LES value of $\left\langle\sigma_{w}\right\rangle_{z / H=1}$ at the UCL top resulted in exchange velocities of $E_{x}=0.07 \mathrm{~m} \mathrm{~s}^{-1}$ and $E_{y}=0.08 \mathrm{~m} \mathrm{~s}^{-1}$. These agree well with $E_{x}=E_{y}=0.09 \mathrm{~m} \mathrm{~s}^{-1}$ that were obtained via the parametrisation for $\sigma_{w}$ based on $u_{*}$ that is used in SIRANE-1. However, compared to $E_{y}=0.15 \mathrm{~m} \mathrm{~s}^{-1}$ derived from Eq. (7) the SIRANE-1 value of $E_{y}$ is $40 \%$ lower. During the model run, both network models determine the direction of vertical transport for a certain street via the local vertical concentration gradient between UCL and above-roof concentrations. A summary of all exchange velocities is given in Tab. 3 .

\subsubsection{Mixing conditions}

The flux parametrisations in the street-network modelling framework (Eqs. 5 and 6) are based on the assumption that pollutants are well-mixed within each box, i.e. spatial gradients within individual streets are small [6]. The appropriateness of this approximation is examined on the basis of the high-resolution LES data. Figure 10 shows the distribution of spatial root-mean-square (r.m.s) values of concentrations in each network-model box as a fraction of box-averaged concentrations for two layers: $0 \leq z / H \leq 1$ and $1 \leq z / H \leq 2$. The smaller the value of this ratio, the better the mixing within the volume. Not surprisingly, upwind of the source and at the lateral edges of the plume, the well-mixed condition is not satisfied and spatial concentration fluctuations are of the same order or greater than the volume average. Particularly strong gradients are found in and around the source street, whereas only a few streets downwind the pollutants had enough time to become well mixed. Hence, in those plume regions where significant levels of concentrations are encountered (Fig. 7) the street-network dispersion models can be expected to perform best.

Within the UCL, two interesting patterns can be observed: On the one hand, the intersection boxes tend to be less well mixed than the neighbouring street boxes, whereas the short $x$ streets, on the other hand, tend to be better mixed than the surrounding boxes. Both features become more apparent at the plume edges. The patterns can be related to the typical flow behaviour observed in the DIPLOS array as discussed in Sect. 4.1 (see Fig. 4). In the $x$ streets pollutants are trapped within the prevalent recirculating flow and hence become better distributed over the street volume. The intersection flow is strongly three-dimensional and thus more prone to the mixing-in of 'clean' ambient air, which becomes increasingly relevant at the edges of the plume. 


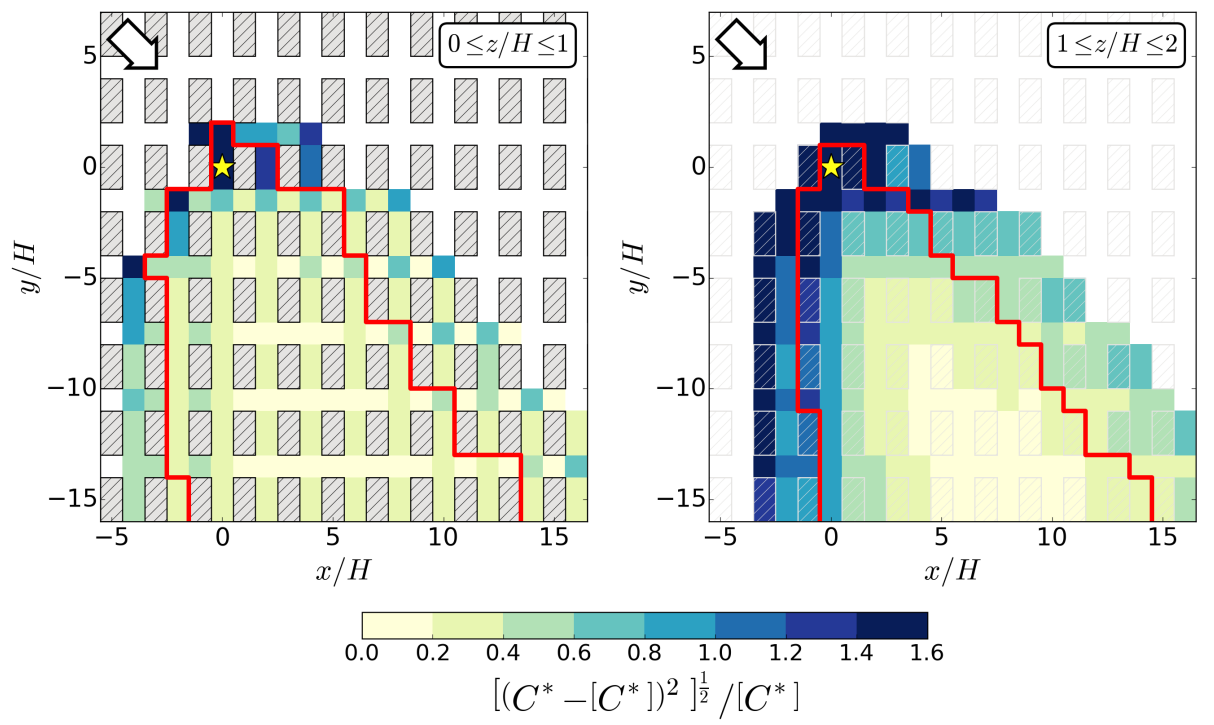

Fig. 10: Spatial r.m.s. values of concentrations in each network-model box as a fraction of box-averaged concentrations within the UCL (left; $0 \leq z / H \leq 1$ ) and in the external layer just above the buildings (right; $1 \leq z / H \leq 2$ ) derived from the LES. Statistics are shown for boxes where $\left[C^{*}\right] \geq 1 \cdot 10^{-4}$. The thick red lines border the part of the plume where the volume-averaged concentrations are $\geq 1 \cdot 10^{-2}$.

\section{Dispersion model evaluation}

The above analyses showed that the DIPLOS geometry represents an interesting test environment for the dispersion models. While still being a strongly idealised setting, the geometric asymmetry together with the existence of a mixture of different flow regimes can pose challenges to fast dispersion models.

In the following, the plume predictions from the different dispersion models introduced in Sect. 3 (Tab. 1) are inter-compared. To provide a suitable benchmark for the intercomparison, concentration data from the LES are used as a reference. The wind-tunnel data are not spatially extensive enough to be used directly for this purpose, but can instead be employed to validate the LES. Indeed, comparison with the statistics from the wind-tunnel model in this study and in previous validation exercises carried out in DIPLOS [23,32] showed that the LES overall represents the salient dispersion features well for this test case, given the uncertainties associated with the simulation and the experiment. While most of the following quantitative comparisons are between the LES and the dispersion models, the experimental data presented in Sect. 4.2 will be revisited for a qualitative appraisal. As we set out in this study to evaluate the street-network modelling approach in comparison to the more established model categories, we need to compare the model results in a common framework. For that, the space-resolved output from the LES, the Gaussian and Lagrangian models is converted into volume-averaged concentrations in boxes covering streets and intersections within the UCL as in the street-network representation. Although this means sacrificing spatial resolution, the assessment of danger zones based on street-integrated con- 
centrations is more practical in emergency-response contexts. Hence, the space-resolution limitation of the street-network modelling is no detriment for this type of application.

\subsection{Qualitative model inter-comparison}

A qualitative inter-comparison of model performances is presented below in terms of concentration footprints and plume characteristics in the DIPLOS canopy. A quantitative assessment of model spreads and biases is given in Sect. 5.2.

\subsubsection{Plume footprints}

Figure 11 compares volume-averaged UCL concentrations, [ $\left.C^{*}\right]$, from all dispersion models with the LES output. A quantitative comparison of these results is shown in Fig. 12 in terms of horizontal transects of volume-averaged concentrations along $x$ ('lateral') and $y$ ('longitudinal') directions and corresponding transects of point-wise concentrations from the experiment in a height of $z / H=0.5$. Due to the different nature of wind-tunnel data compared to the volume averages, these are meant to supplement the qualitative appraisal of the plume patterns.

The decisive difference between the Gaussian models concerns the added plume deflection, either based on the above-rooftop flow (GAUSS -1 ; plume centreline along $-54^{\circ}$ ) or on the representative UCL wind direction (GAUSS $-2 ;-78^{\circ}$ ). The latter clearly resulted in a better agreement with the LES and also with the footprint in the wind tunnel at half the building height (Fig. 7). The strong lateral plume spread is governed by the enhancement term for $\sigma_{y}$ (Eq. 2) for light-wind situations. Not considering this modification of the classic Briggs formulation results in too narrow plumes and a significantly poorer agreement with the LES (not shown). Naturally, the Gaussian models do not capture topological dispersion effects like the strong pollutant channelling into $-y$ direction and the uneven splitting in intersections, which resulted in the asymmetric plume shape. The symmetry constraint leads to too strong upwind spread into $-x$ direction in GAUSS -2 and hence a much broader plume in the far-field $(\Delta x \simeq 22 H)$ compared to the LES $(\Delta x \simeq 18 H)$ at $y / H=-13.5$ (Fig. 12). The best quantitative agreement with the LES is found farther away from the source in those downwind regions of the plume where material is well-mixed within and above the canopy (Fig. 10) and where the magnitude of vertical concentration fluxes at the canopy top is small (Fig. 9a).

A much better overall agreement with the plume shape of the LES and the wind tunnel is found in the outputs of the Lagrangian model. Some differences can be observed in the runs based on the two native QUIC flow modules, QUIC (URB) and QUIC (CFD). In the former, a strong lateral spread of the plume into $-x$ streets is observed, which close to the source is comparable to GAUSS -2 (see $y / H=-4.5$ transect in Fig. 12). This behaviour can be attributed to the stronger negative $u$-component of the horizontal flow observed in the QUIC-URB wind fields (see Figs. 4 and 5), which leads to a redistribution of material from the intersections into the upwind short streets, there entering the large recirculation zone. It is noted that there are no data available from the wind-tunnel campaign to further investigate the spread of the plume into $-x$ direction. The downstream extent $(+x)$ of the plume and the distribution of scalars along the $x / H=0$ transects through the source street, however, agree well with the reference data.

Although the flow field from the RANS model used in QUIC (CFD) in large part showed a flow channelling along the $y$ streets and through the intersections similar to the turbulence- 


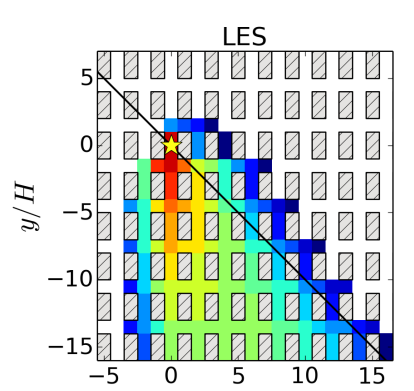

QUIC (URB)
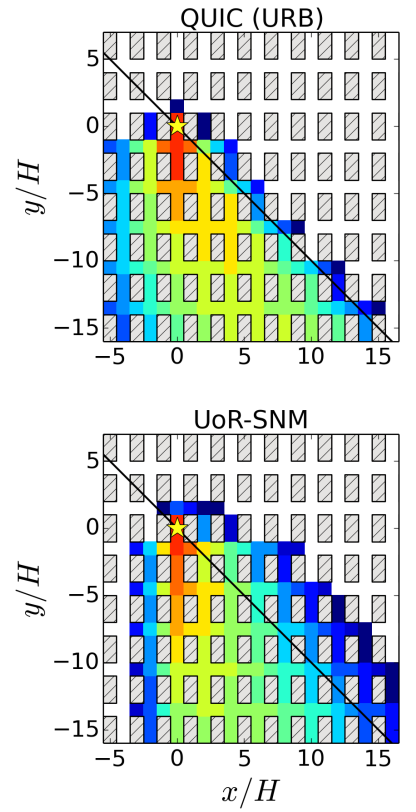

GAUSS-1

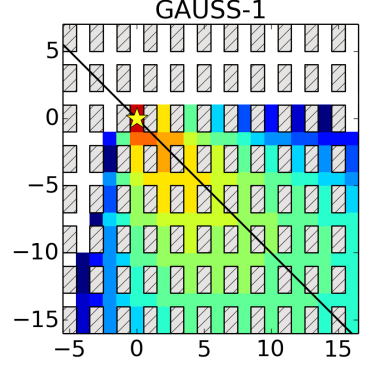

QUIC (CFD)

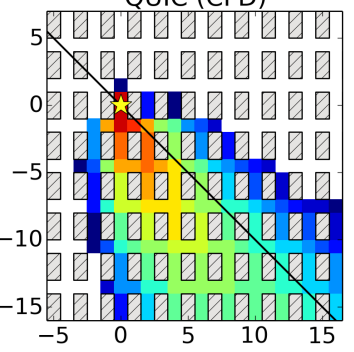

SIRANE-1

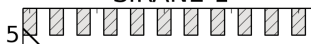

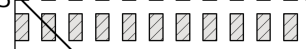

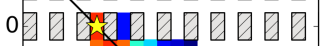

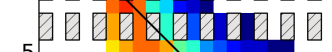

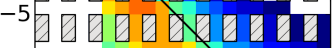

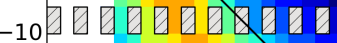
月时日时日大

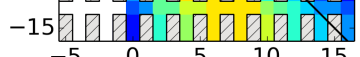

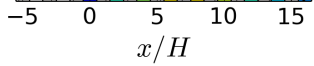

GAUSS-2

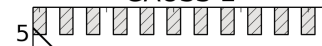

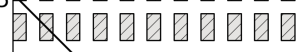

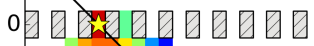

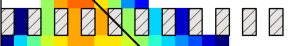

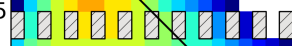

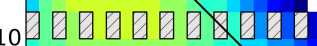

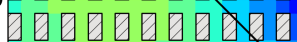

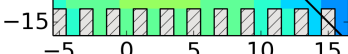

OUIC (LES)

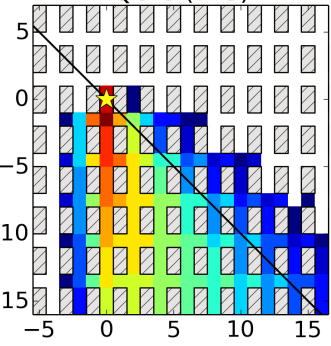

SIRANE-2

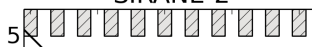

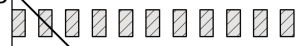

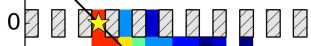

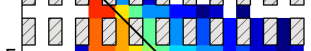

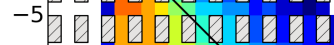

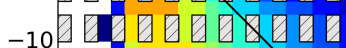
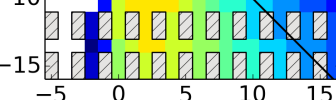

$\begin{array}{llllll}-5 & 0 & 5 & 10 & 15\end{array}$

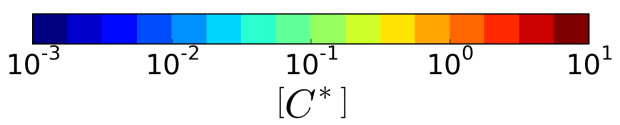

Fig. 11: Volume-averaged concentrations in streets and intersections within the canopy layer $(0 \leq z / H \leq 1)$ for the LES reference data and the different dispersion models. The solid black line indicates the forcing wind direction of $-45^{\circ}$.

resolving LES, the resulting plume orientations are somewhat different. QUIC (CFD) has stronger transport of material into $+x$ direction than the LES as a result of the stronger outflow from the intersections into the downwind short streets (Fig. 4, Tab. 3). The qualitative comparison with the point-wise concentrations from the wind tunnel also shows an underprediction along the $x / H=0$ transect, but a better agreement at some distance away from the source $(y / H=-13.5)$. QUIC-PLUME was also run on turbulence fields provided directly by the RANS turbulence model. This resulted in similar results to the QUIC (CFD) output presented here, but with a slightly reduced lateral plume spread (not shown). 

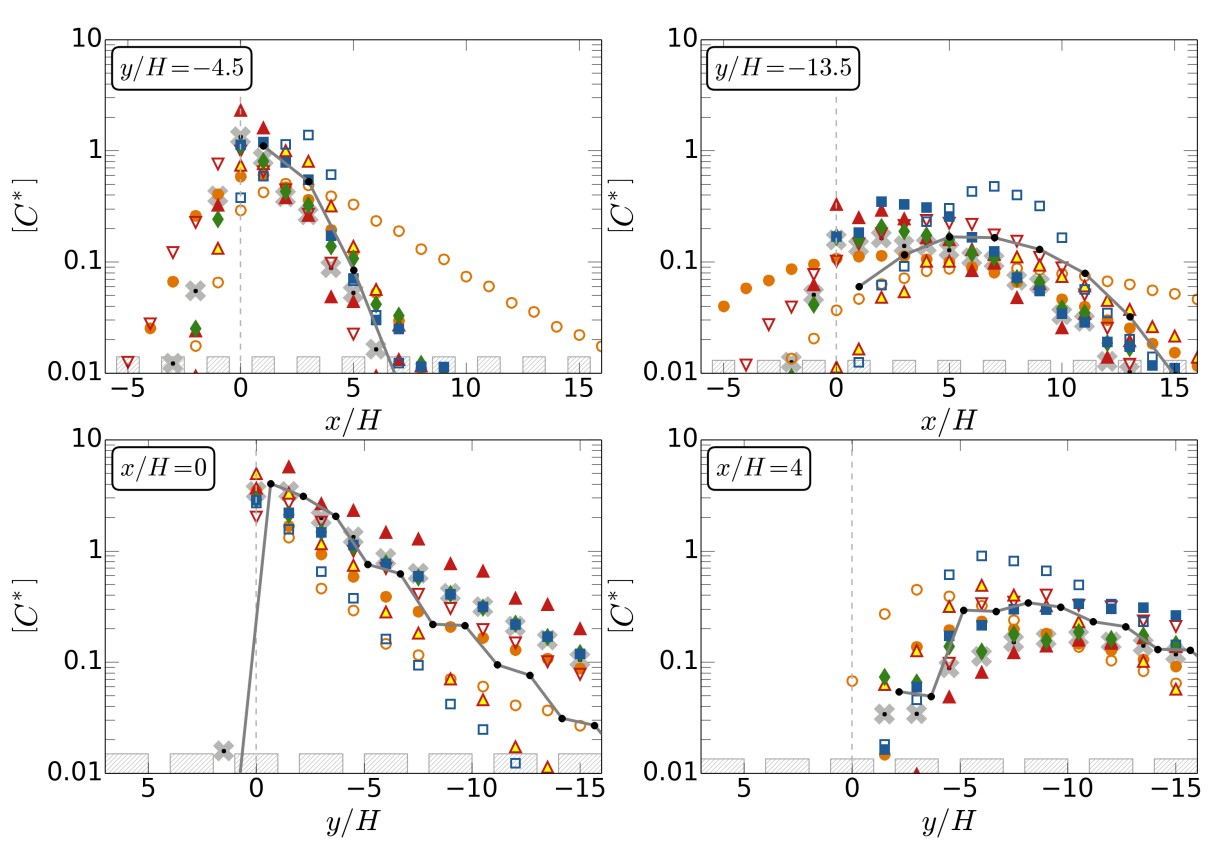

\begin{tabular}{|c|c|c|c|c|c|c|c|c|c|}
\hline$*$ & LES & 0 & GAUSS-1 & $\nabla$ & QUIC (URB) & $\Delta$ & QUIC (LES) & 口 & SIRANE-1 \\
\hline$\rightarrow$ & WT & - & GAUSS-2 & $\Delta$ & QUIC (CFD) & - & UoR-SNM & - & SIRANE-2 \\
\hline
\end{tabular}

Fig. 12: Comparison of horizontal transects of volume-averaged concentration along the $x$-axis (top) and along the $y$-axis (bottom) in the canopy layer. Single-point measurements from the wind tunnel (WT) at a height of $z / H=0.5$ are shown as well for the sake of completeness. The source position of $x / H=y / H=0$ is indicated by a dashed vertical line.

Not surprisingly, the best QUIC-PLUME agreement with the LES is found for the QUIC (LES) set-up. Differences apparent here are only attributable to the Lagrangian dispersion modelling component, which in this case demonstrates the suitability of the QUIC-PLUME urban dispersion algorithms. The largest deviations are apparent along the plume centreline, where the model over-predicts concentration levels as seen in the longitudinal transect at $x / H=0$ in Fig. 12 compared to the Eulerian solution from the LES and the experimental data. This is paralleled by a slightly larger lateral spread $(+x)$ of the plume compared to QUIC (URB) and QUIC (CFD). We also observe that QUIC (LES) is the only model where the maximum volume-averaged concentration is not located in the source street, but in the first downwind intersection box.

As expected, the street-network model UoR-SNM run on LES velocity parameters matches the longitudinal and lateral concentration profiles computed by the LES extremely well. This demonstrates that, despite the minimal flow specifications needed, the simple fluxbalance methodology is suitable for capturing important features of canopy-layer dispersion. This is largely attributable to the fact that the model formulation explicitly represents the street topology and directly accounts for associated topological dispersion effects. Running UoR-SNM with the re-entrainment term switched on and off is helpful to reveal the significance of adequately representing the vertical pollutant fluxes. This analysis showed that in regions where re-entrainment dominates (see Fig. 9a), volume-averaged UCL concen- 

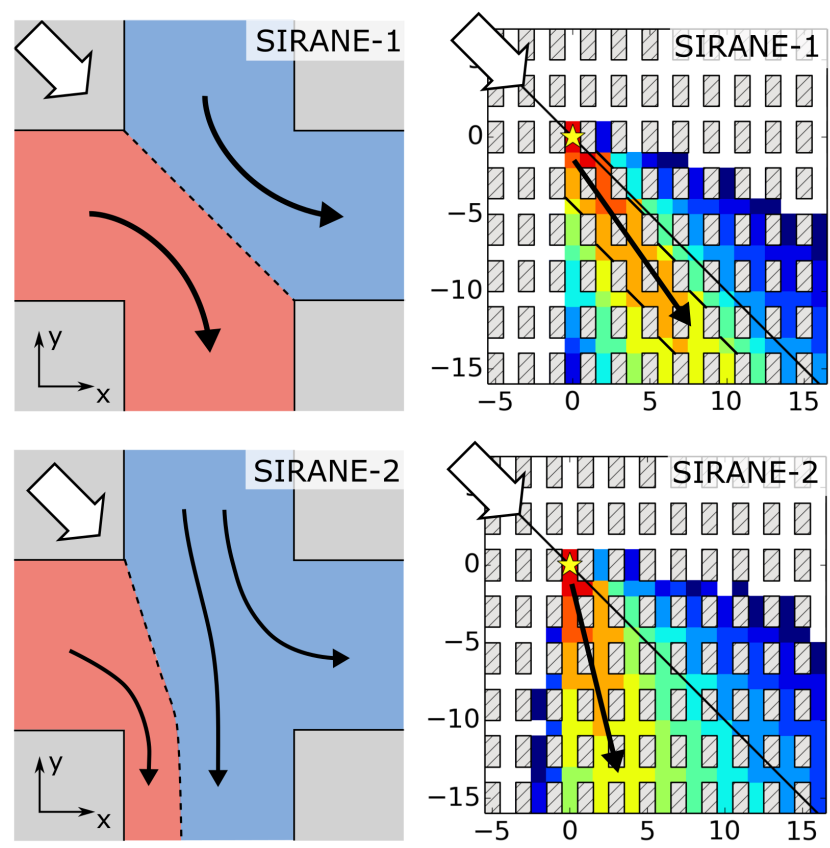

Fig. 13: Schematic of the flux distribution in the intersections for SIRANE-1 with $\left\langle U_{s}\right\rangle=$ $\left\langle V_{s}\right\rangle$ predicted by the SIRANE flow model (top left), and SIRANE-2 with $\left\langle U_{s}\right\rangle \simeq 0.3\left\langle V_{s}\right\rangle$ based on the LES information (bottom left) together with the corresponding canopy-layer plume footprints and the mean plume advection direction. The forcing wind direction is indicated by thick arrows.

trations can be enhanced by an order of magnitude or more (not shown). As evident from comparing Figs. 9a and 11, the re-entrainment regions in the LES feature non-negligible concentration levels in agreement with the experiment (Fig. 7). The strongest deviations between the demonstration model UoR-SNM and the LES occur very close to the source and at the plume edges, where the well mixed-condition breaks down.

SIRANE-1, which was run in operational mode with parametrisations for horizontal advection and vertical exchange velocities, predicts a plume orientation that is much closer to the $-45^{\circ}$ forcing wind direction than any of the other models. As anticipated in Sect. 4.1.1, the larger $\left\langle U_{s}\right\rangle$ computed the SIRANE-1 flow model resulted in enhanced advection along the short $x$ streets as compared to the LES, which also affected the distribution of material from the intersection into the downwind streets. Overall, the plume is less well diluted farther away from the source than in the LES or in the experiment $(y / H=-13.5$ and $x / H=4$ transects in Fig. 12). Unlike SIRANE-2, for which $\left\langle U_{s}\right\rangle\left\langle\left\langle V_{s}\right\rangle\right.$ resulted in an uneven branching of the plume in the intersection, in SIRANE-1 the material is uniformly distributed into the downwind streets since $\left\langle U_{s}\right\rangle=\left\langle V_{s}\right\rangle$. The observed deviation from the $-45^{\circ}$ forcing direction is induced by the rectangular shape of the buildings.

This behaviour is schematically illustrated in Fig. 13. As a consequence the models predict considerably different plume orientations. The even plume splitting in the intersections in SIRANE-1 also led to a reduced lateral spread of pollutants. This spatial confinement of material together with the reduced vertical exchange velocities compared to SIRANE-2 
(Tab 3) is responsible for the significant over-prediction of concentration levels compared to the volume-averaged LES and the point-wise wind-tunnel data. SIRANE-2, which was provided with representative velocities and hence accounts for the dominance of pollutant flux down the $y$ streets from the intersections, shows a high level of agreement with the LES.

\subsection{Quantitative model inter-comparison}

In order to quantify the differences between the dispersion models and the LES, we use a set of well-established dimensionless validation metrics $[24,13,40]$. These are the factor of two of observations (FAC2), the fractional bias (FB), the normalised root mean square error (NMSE), the geometric mean bias (MG), geometric variance (VG) and the correlation coefficients $(\mathrm{R})$ as defined in Eqs. 11-16. As for the qualitative comparison, the quantification of differences between the LES, $C_{o}$, and the model predictions, $C_{p}$, is conducted in terms of a data-pairing of non-dimensionalised, box-averaged UCL concentrations, $\left[C^{*}\right]$. Curly brackets, $\{\ldots\}$, indicate the average over the entire data sample of $N$ box-averaged concentrations and $\sigma_{C}$ are the corresponding sample standard deviations.

Factor of two:

$$
\text { FAC2 }=\frac{1}{N} \sum_{i} F_{i} \text { with } F_{i}= \begin{cases}1, & \text { if } \frac{1}{2} \leq \frac{C_{p, i}}{C_{o, i}} \leq 2 \\ 0, & \text { otherwise }\end{cases}
$$

Fractional bias:

$$
\mathrm{FB}=2 \frac{\left(\left\{C_{o}\right\}-\left\{C_{p}\right\}\right)}{\left(\left\{C_{o}\right\}+\left\{C_{p}\right\}\right)}
$$

Normalised mean square error:

$$
\mathrm{NMSE}=\frac{\left\{\left(C_{o}-C_{p}\right)^{2}\right\}}{\left\{C_{o}\right\}\left\{C_{p}\right\}}
$$

Geometric mean bias:

$$
\mathrm{MG}=\exp \left(\left\{\ln C_{o}\right\}-\left\{\ln C_{p}\right\}\right)
$$

Geometric variance:

$$
\mathrm{VG}=\exp \left(\left\{\left(\ln C_{o}-\ln C_{p}\right)^{2}\right\}\right)
$$

Correlation coefficient:

$$
\mathrm{R}=\frac{\left\{\left(C_{o}-\left\{C_{o}\right\}\right)\left(C_{p}-\left\{C_{p}\right\}\right)\right\}}{\sigma_{C_{o}} \sigma_{C_{p}}}
$$

Fig. 14 shows the underlying scatter plots for the LES and the dispersion models, as well as a comparison of point-values at $z / H=0.5$ from the LES and the wind tunnel to complement the earlier qualitative comparison in Fig. 7. Here we used a nearest-neighbour approach to match the LES data to the exact measurement locations and heights of individual wind-tunnel data points.

As discussed in detail by Chang and Hanna [24], in order to obtain a comprehensive picture about the model, different validation metrics should be consulted together. While FB and MG measure the systematic bias of the model and can be influenced by cancelling errors, NMSE and VG measure the mean relative scatter between the data pairs and include systematic and random errors. By using a logarithmic framework, MG and VG are 
less susceptible to infrequently occurring very high or low concentrations than their 'linear' counterparts, FB or NMSE. This is beneficial in test cases such as the one in this study, where results are compared over several decades of concentrations. $\mathrm{R}$ is not a reliable indicator of model accuracy since it is dominated by the fact that concentrations will generally decrease with distance from the source [24]. However, it provides information about the level of common variation in both data sets and can be useful in combination with the other metrics. FAC2 and FAC5 provide the most robust measure with regard to the influence of isolated events of very good or bad agreement between data pairs.

FAC2, FAC5, FB, NMSE and R were obtained from data pairs for which either the LES or the model output was $\geq 1 \cdot 10^{-3}$ so that misses and false positives are reflected in the metrics. This is not as easy for the MG and VG metrics as these can be overly affected by very low concentration values and are undefined for zero concentrations (plume misses a street completely). For these metrics we follow the recommendation by Chang and Hanna [24] and impose a minimum threshold of $\left[C^{*}\right]=1 \cdot 10^{-3}$ on all data.

Table 4 lists the metrics together with the target values for a model that perfectly matches the LES. As a point of reference for the assessment of urban dispersion models, Chang and Hanna [24] and later Hanna and Chang [40] have proposed the following acceptance criteria for a 'good' model performance: FAC2 $>0.3$ (or $>0.5$ based on earlier assessments), $|\mathrm{FB}|<$ 0.67 , NMSE $<6,0.7<M G<1.3$ and $V G<1.6$. In other words, the mean model bias as measured by FB and MG should be within $30 \%$ of the mean and the mean relative scatter (NMSE and VG) within approximately a factor of 2 of the mean. It has to be noted that these acceptance thresholds were originally proposed for arc-maximum concentrations, but meanwhile are also commonly applied to assess the model performance over the entire extent of the plume. In general, however, it is important to highlight that such thresholds should be understood as being strongly case-specific and linked to the margins of error that are acceptable in the scenario under investigation. In the absence of such constraints in this study, we revert to the criteria proposed by Hanna and Chang [40].

Only GAUSS-2, QUIC (URB), QUIC (LES) and UoR-SNM are within a factor of 2 of the LES more than $30 \%$ of the time. Of these, QUIC (URB) is the only model that was not provided with information from the LES flow. Only QUIC (LES) and UoR-SNM meet the less stringent FAC5 criterion more than $75 \%$ of the time. For both FAC2 and FAC5, SIRANE-1 persistently shows the lowest values. In contrast to that, the overall low FB indicates only small systematic bias in all models. Inspecting the corresponding scatter plots in Fig. 14, however, shows that in some cases this is a result of error cancellation of over and under-predictions. This is particularly apparent in the plots for QUIC (CFD) and SIRANE-1, where data pairs group symmetrically about the 1-to-1 line. According to the MG metric, all models except for QUIC (LES) which is closest to the ideal value of 1.0, have a tendency to over-predict mean concentrations (positive bias). The strongest deviations from the LES reference are associated with approximately a factor of 2 mean over-prediction as seen for the Gaussian models $(\mathrm{MG} \simeq 0.5)$. The VG metric shows the highest relative scatter with almost a factor of 7 of the mean for GAUSS-1 as a result of the largest mismatch of plume footprints. QUIC (CFD) and SIRANE-1, which showed a similar tendency in the plume centreline although associated with different concentration levels, have comparable VG values indicating a relative scatter of about a factor of 4. QUIC (URB), QUIC (LES) and UoR-SNM exhibit the smallest scatter. This is also reflected in very high correlation coefficients compared to GAUSS-1 and SIRANE-1.

Parts of the above results are visually summarised in a Taylor diagram [76] in Fig. 15, based on the normalised standard deviation, $\sigma_{C_{p}} / \sigma_{C_{o}}$, the normalised relative root-mean- 

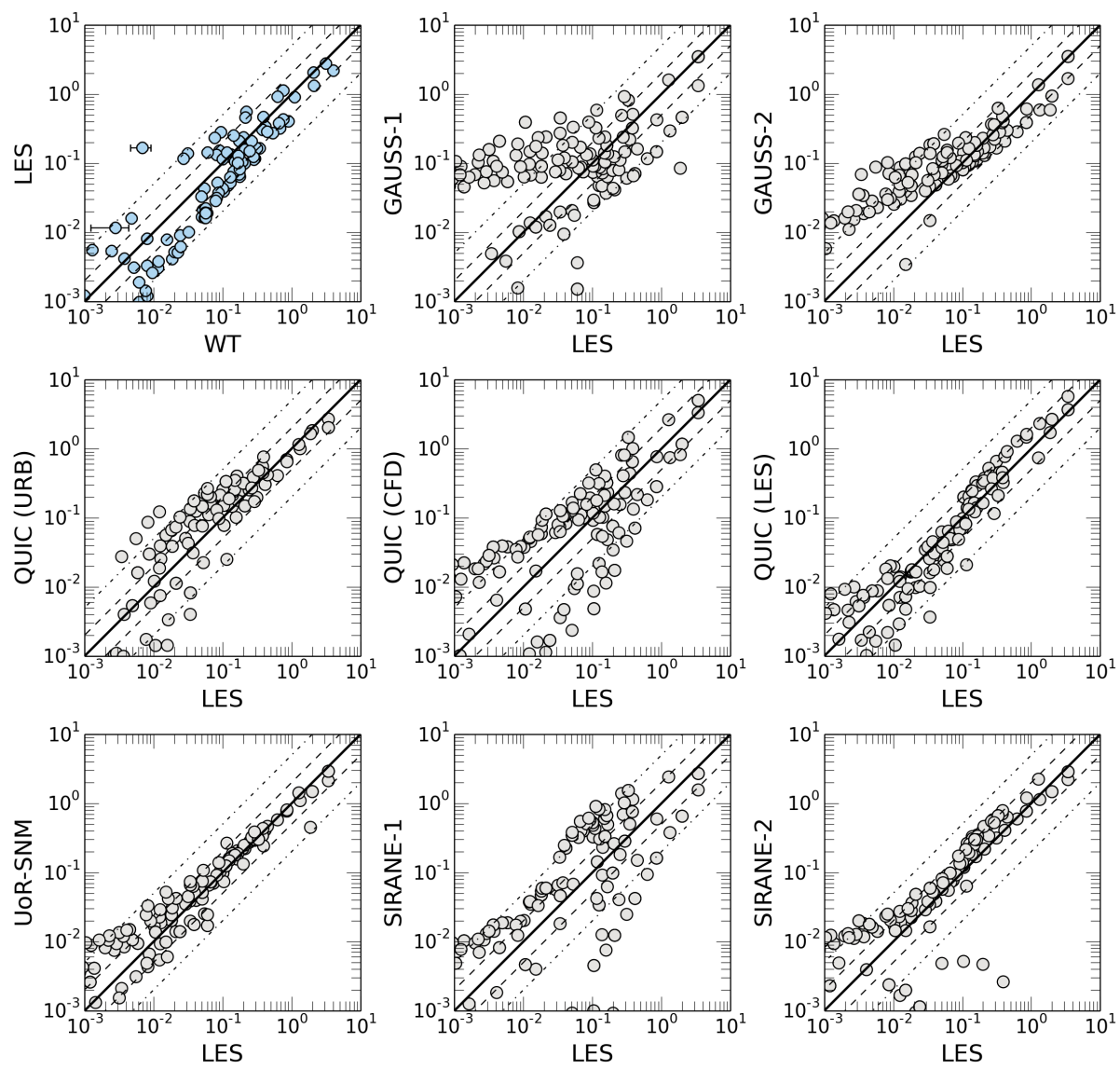

Fig. 14: Scatter plots of model predictions versus the LES based on volume-averaged UCL concentrations $[C]^{*}$. In contrast to that, the upper left plot shows LES and wind-tunnel singlepoint data pairs at a nominal height of $z / H=0.5$ (see plumes in Fig. 7). Thick solid lines indicate the ideal 1-to-1 relationship; dashed and dashed-dotted lines show the factor-of-2 and factor-of-5 margins, respectively.

square error and the correlation coefficient, which all measure the random (non-systematic) scatter are related to each other through the law of cosines [24].

The diagram shows a cluster of models that have a high level of agreement with the LES (GAUSS-2, QUIC (URB), SIRANE-2 and UoR-SNM) with comparably low rootmean-square errors $(\sim 0.4)$ and high correlation $(0.9-0.96)$, but overall smaller variability compared to the LES reference $\left(\sigma_{C_{p}} / \sigma_{C_{o}}<1\right)$. SIRANE-1 and GAUSS-1 show comparable metrics with larger random errors than the other models. Only the output from the Lagrangian dispersion runs based on the CFD-RANS and LES wind fields overall exhibit a larger variability than the LES, which could be related to the fact that the material is diluted over a larger lateral region than in the LES or QUIC (URB). An interesting observation is that QUIC (URB) agrees better with the LES than QUIC (LES), although the latter is run on the LES mean flow fields that showed some significant differences to the flow pattern from 
Table 4: Evaluation metrics for all models in comparison to the LES reference data. All metrics were computed from box-averaged concentrations $\left[C^{*}\right]$ within the UCL. The target values in the sense of a perfect agreement with the LES are given together with the maximum box-averaged concentrations in the domain, $\left[C^{*}\right]_{\max }$, from all data sets.

\begin{tabular}{|c|c|c|c|c|c|c|c|c|}
\hline Model & {$\left[\mathbf{C}^{*}\right]_{\max }$} & FAC2 & FAC5 & FB & NMSE & MG & VG & $\mathbf{R}$ \\
\hline Target value & - & 1.0 & 1.0 & 0.0 & 0.0 & 1.0 & 1.0 & 1.0 \\
\hline LES & 3.42 & - & - & - & - & - & - & - \\
\hline GAUSS-1 & 3.47 & 0.21 & 0.42 & 0.07 & 4.11 & 0.54 & 38.44 & 0.76 \\
\hline GAUSS-2 & 3.49 & 0.37 & 0.60 & 0.13 & 1.89 & 0.56 & 3.70 & 0.90 \\
\hline QUIC (URB) & 2.69 & 0.44 & 0.71 & -0.06 & 0.71 & 0.73 & 2.46 & 0.96 \\
\hline QUIC (CFD) & 4.99 & 0.23 & 0.55 & -0.11 & 1.95 & 0.85 & 7.92 & 0.89 \\
\hline QUIC (LES) & 5.71 & 0.58 & 0.86 & -0.26 & 1.46 & 1.02 & 1.66 & 0.96 \\
\hline UoR-SNM & 2.91 & 0.60 & 0.83 & 0.12 & 1.06 & 0.80 & 1.60 & 0.96 \\
\hline SIRANE-1 & 2.70 & 0.11 & 0.34 & -0.27 & 5.10 & 0.61 & 7.27 & 0.65 \\
\hline SIRANE-2 & 2.87 & 0.29 & 0.42 & -0.23 & 2.41 & 0.74 & 3.79 & 0.86 \\
\hline
\end{tabular}

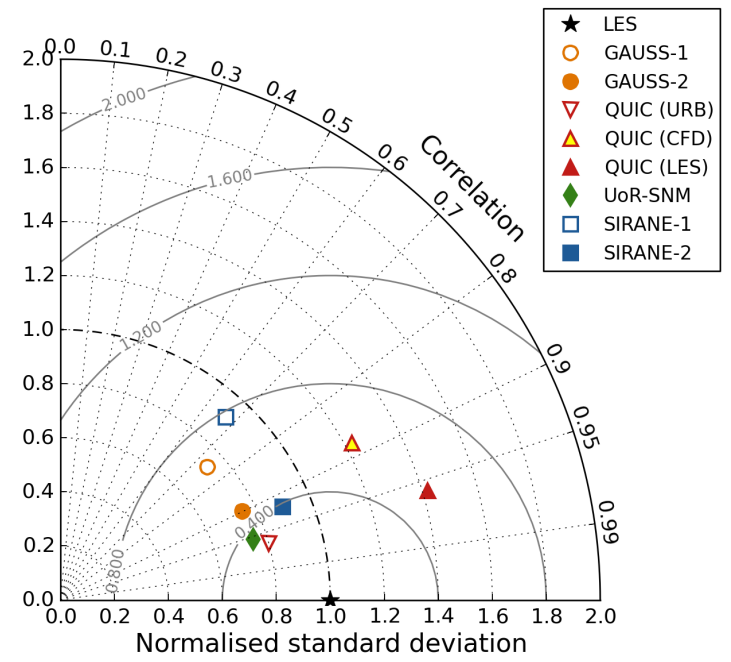

Fig. 15: Taylor diagram based on the normalised standard deviation (dotted arcs), the normalised relative root-mean-square error (solid arcs) and the correlation coefficient $\mathrm{R}$ (cosine of the angle to the horizontal axis; dotted lines). The thick dashed arc indicates $\sigma_{C_{p}} / \sigma_{C_{o}}=1$. The star symbol shows the LES reference.

QUIC-URB (Fig. 4). Given the differences in the concentration fields, this implies that in QUIC (URB) the component of flow reversal $(-x)$ counteracts the tendency of the dispersion module to produce a stronger downwind spread $(+x)$ of the plume. Interestingly, among the well-informed models, the performance is not directly correlated with the amount of flow information provided. Although QUIC (LES) was run on data of the entire high-resolution LES mean flow, the Lagrangian model did not outperform the much simpler Gaussian and street-network models that were only provided with few velocity parameters. 


\section{Further discussions and conclusions}

We presented a process-based evaluation of different methods for fast urban dispersion modelling for emergency-response applications. The focus was put on the comparison of UCL concentration footprints resulting from the continuous release of pollutants from a ground source. Representatives across the hierarchy of dispersion modelling approaches were evaluated: (i) Gaussian and (ii) Lagrangian models and the comparatively new (iii) street-network modelling. The urban test bed, albeit with geometric simplicity, induced complex mean flow patterns that resulted in a strong plume asymmetry. Capturing the resulting topological dispersion features proved to be a challenge for the models tested.

Running the models in different configurations with respect to the detail of flow information provided, resulted in large differences in performance when compared to data from high-resolution LES. The strongest effect was seen in the two simplest modelling categories: the Gaussian and the street-network models. The simple baseline Gaussian plume model used in this study improved significantly after some degree of building-awareness was added by means of a plume deflection in the UCL. However, the geometry-induced asymmetry of the plume and other topological dispersion features cannot be captured as there is no explicit awareness of the urban morphology in this model class. It is emphasised, however, that more advanced Gaussian dispersion models are available as outlined in Sect. 1.2, some of which have added capabilities to take into account bulk effects of typical street-canyon flow and validation studies of such Gaussian plume or puff models can be found in the literature, e.g. $[16,60]$.

Running the street-network model UoR-SNM on flow parameters completely derived from the reference LES provided a demonstration of the suitability of the street-network methodology for canopy-layer dispersion modelling, and showed that the main relevant dispersion processes were captured. Advective transport mechanisms like pollutant channelling along streets and plume splitting in intersections were adequately represented by flux-balance parametrisations, just as the vertical turbulent transfer of pollutants between UCL and external boundary layer. Naturally, detailed flow information is not usually available in an emergency event. Hence, operational urban dispersion models have to rely on suitable parametrisations of relevant building-induced flow features.

Lagrangian models require the largest amount of input information in terms of 3D mean flow fields that need to be provided by an external module. Running QUIC-PLUME offline on flow fields from three different building-resolving simulations (diagnostic, CFD-RANS and LES) highlighted the strong dependence of the dispersion pattern on the underlying flow structure. The work also highlighted the benefits of conducting basic process studies like this in idealised geometries. In the DIPLOS array processes are complex enough to be challenging for models, while it is still possible to understand causalities. Initial runs with the diagnostic QUIC-URB model for this study, for example, revealed bugs in the flow module, which had a strong effect on the plume dispersion behaviour. Once identified, these bugs were easily corrected by the developers. Such errors would have been much harder to detect in more complex geometrical settings where it can be difficult to distinguish genuine features from artefacts.

\subsection{Run-speed requirements}

Regarding computing times, the two street-network dispersion models and the Gaussian model performed comparably with run speeds of $\mathscr{O}(1 \mathrm{~min})$ on a typical desktop computer. 
We ran all QUIC simulations in parallel on two cores on a Windows computer with an Intel Core i5 3.3 GHz processor. This resulted in run times for the QUIC-URB flow module of approx. $1 \mathrm{~min}$ and of $\sim 1 \mathrm{~h}$ for the QUIC-CFD RANS model. Additional computing times from the Lagrangian stochastic model also were in the order of $1 \mathrm{~h}$. However, it is emphasized that the QUIC-PLUME set-up used in this study was designed for the purpose of an evaluation exercise and not for operational use. Much faster computing times in complex urban environments of $\mathscr{O}(1 \mathrm{~min})$ to $\mathscr{O}(10 \mathrm{~min})$ can be achieved with QUIC-PLUME in general and for the DIPLOS geometry in particular by running on more cores and using an optimised combination of fewer particles, larger model time steps and shorter averaging periods [M. Brown, pers.comm.]. Current advancements of the QUIC system focus on the optimisation of computational speed by running on graphics processors $[66,64]$. Based on these studies, it is likely that the new GPU-PLUME model can run up to 180 times faster than QUIC-PLUME for typical urban dispersion scenarios.

\subsection{Strengths and limitations of the street-network approach}

One aim of this study was to assess the performance of street-network models against more established methods based on an idealised test case with a building packing density representative of city centres. While requiring much fewer velocity input parameters, in the idealised-geometry scenario investigated here the simple street-network models performed equally well or better compared to the more complex Lagrangian dispersion model run on full 3D wind fields, when compared to data from the high-resolution, turbulence-resolving LES. At the same time, computational costs and computing times associated with the network approach are low. Unlike the similarly inexpensive Gaussian plume models, streetnetwork models directly account for building-induced dispersion effects. We showed that the conceptual design of models like SIRANE and UoR-SNM enables to represent the dominant processes affecting pollutant dispersion in the DIPLOS canopy: topological dispersion effects like channelling along streets and branching at intersections as well as pollutant exchange with the external flow.

The basic rationale behind the approach is to study urban dispersion at the scales of interest for emergency-response applications: entire street canyons and intersections. For the regular, equal-height DIPLOS geometry, we could show that such a volume-averaged representation of concentrations becomes representative after a short distance from the source and particularly in those regions of the plume where concentration levels are non-negligible. However, the spatial variability of the mean concentration patterns is expected to be enhanced in the case of non-stationary wind forcing as encountered in the natural atmosphere. Naturally this model formulation is also associated with uncertainties regarding the exact location of emission sources and receptors within a street segment. The location of the source with regard to the surrounding buildings and the prevailing flow patterns can have a strong influence on the near-field dispersion behaviour. In scenarios involving very long streets a too coarse resolution has to be avoided by subdividing into shorter segments.

The study highlighted the importance of flow-field modelling in all types of operational dispersion models. Whether or not the driving flow is representative of the encountered scenario to a large degree determines the prediction quality that can be achieved with the dispersion model. An evaluation of the flow parametrisations in SIRANE showed a dependence of the accuracy of modelled horizontal advection velocities on the length of the street. In short streets the modelling assumption of a fully developed flow field does not apply, which resulted in an over-prediction of along-street velocities in the current test case. This 
had knock-down effects on the way in which material is redistributed from the intersection into the downwind streets. Not capturing the uneven branching in the intersections of the DIPLOS array resulted in significant differences between plume centrelines in SIRANE-1 and the LES. Related to the advection characteristics in the UCL is the representation of deviations from the forcing wind direction in the roughness sublayer above the buildings. The current SIRANE parametrisation of the vertical turbulent exchange velocity based on $u_{*}$ does not account for local mixing effects in the roughness sublayer above the buildings and hence is not a complete way of representing this process.

Further limitations of the street-network approach are expected to result from the fact that SIRANE and UoR-SNM were developed for street-canyon dispersion in urban environments with high packing density, where there is a sufficient degree of decoupling between UCL and the external boundary layer. On the city-scale, however, urban environments are comprised of areas with vastly different morphological characteristics, for some of which the street-network modelling framework breaks down. For dispersion through 'open' areas like parks or squares, through very wide streets (wake interference or isolated roughness regimes) or streets only partially bordered by buildings different processes need to be considered and parametrised. Additionally, the need to account for environments with a significant heterogeneity of building heights is an area of ongoing model development. Furthermore, studying effects of atmospheric stratification (stable, unstable) on urban dispersion and their parametrisation in street-network models have become a priority for further experimental and computational work.

\section{Acknowledgements}

The DIPLOS project is funded by the UK's Engineering and Physical Sciences Research Council grants EP/K04060X/1 (Southampton), EP/K040731/1 (Surrey) and EP/K040707/1 (Reading). The EnFlo wind tunnel is an NCAS facility and we gratefully acknowledge ongoing NCAS support. We are grateful for comments and ongoing discussions with other colleagues at Surrey, Southampton and elsewhere. We thank Michael Brown and Eric Pardyjak for providing access to the QUIC dispersion modelling system and helpful discussions throughout this study. Stephen Belcher and Elisa Goulart are gratefully acknowledged for their development of and support with the University of Reading Street-Network Model (UoR-SNM). The wind-tunnel data measured in the DIPLOS project are available from the University of Surrey (DOI: https://doi.org/10.6084/m9.figshare.5297245). The LES data analysed in this study can be obtained from the University of Southampton (DOI: https://doi.org/10.5258/SOTON/D0314).

\section{References}

1. Allen, C.T., Haupt, S.E., Young, G.S.: Source Characterization with a Genetic Algorithm-Coupled Dispersion-Backward Model Incorporating SCIPUFF. J. Appl. Meteorol. Clim. 46(3), 273-287 (2007)

2. Andronopoulos, S., Armand, P., Baumann-Stanzer, K., Herring, S., Leitl, B., Reison, T., Castelli, S.T. (eds.): Background and Justification Document. COST Action ES1006. University of Hamburg, Germany (2012)

3. Antonioni, G., Burkhart, S., Burman, J., Dejoan, A., Fusco, A., Gaasbeek, R., Gjesdal, T., Jäppinen, A., Riikonen, K., Morra, P., Parmhed, O., Santiago, J.: Comparison of CFD and operational dispersion models in an urban-like environment. Atmos. Environ. 47, 365-372 (2012)

4. Baumann-Stanzer, K., Castelli, S.T., Stenzel, S. (eds.): Model Evaluation Case Studies. COST Action ES1006. University of Hamburg, Germany (2015) 
5. Belcher, S.E.: Mixing and transport in urban areas. Philos. T. Roy. Soc. A 363(1837), 2947-2968 (2005)

6. Belcher, S.E., Coceal, O., Goulart, E.V., Rudd, A.C., Robins, A.G.: Processes controlling atmospheric dispersion through city centres. J. Fluid Mech. 763, 51-81 (2015)

7. Bentham, T., Britter, R.: Spatially averaged flow within obstacle arrays. Atmos. Environ. 37(15), 20372043 (2003)

8. Berkowicz, R.: OSPM - A Parameterised Street Pollution Model. In: R.S. Sokhi, R. San José, N. Moussiopoulos, R. Berkowicz (eds.) Urban Air Quality: Measurement, Modelling and Management: Proceedings of the Second International Conference on Urban Air Quality: Measurement, Modelling and Management; Technical University of Madrid 3-5 March 1999, pp. 323-331. Springer Netherlands, Dordrecht (2000)

9. Biltoft, C.: Customer report for Mock Urban Setting Test. Tech. Rep. Report WDTC-FR-01-121, US Army Dugway Proving Ground, Dugway (UT), USA (2001)

10. Boris, J., Patnaik, G., Obenschain, K.: The how and why of Nomografs for CT-Analyst. Report NRL/MR/6440-11-9326, Naval Research Laboratory, Washington (DC), USA (2011)

11. Boris, J.P.: The threat of chemical and biological terrorism: Preparing a response. Comput. Sci. Eng. 4, 22-32 (2002)

12. Briggs, G.: Diffusion estimation for small emissions. Report ATDL Report No. 79, ATDL, NOAA/ARL, Oak Ridge (TN), USA (1973)

13. Britter, R., Schatzmann, M. (eds.): Model evaluation guidance and protocol document. COST Action 732. University of Hamburg, Germany (2007)

14. Britter, R.E., Hanna, S.R.: Flow and dispersion in urban areas. Annu. Rev. Fluid Mech. 35, 469-496 (2003)

15. Brixey, L.A., Heist, D.K., Richmond-Bryant, J., Bowker, G.E., Perry, S.G., Wiener, R.W.: The effect of a tall tower on flow and dispersion through a model urban neighborhood Part 2. Pollutant dispersion. J. Environ. Monit. 11, 2171-2179 (2009)

16. Brook, D., Felton, N., Clem, C., Strickland, D., Griffiths, I., Kingdon, R., Hall, D., Hargrave, J.: Validation of the Urban Dispersion Model (UDM). Int. J. Environ. Pollut. 20(1-6), 11-21 (2003)

17. Brown, M., Gowardhan, A., Nelson, M., Williams, M., Pardyjak, E.: QUIC transport and dispersion modelling of two releases from the Joint Urban 2003 field experiment. Int. J. Environ. Pollut. 52(3-4), 263-287 (2013)

18. Brown, M.J.: Urban dispersion - challenges for fast response modeling. In: Proceedings of the 5th AMS Symposium on the Urban Environment, p. 13. Vancouver, Canada (2004)

19. Carpentieri, M., Hayden, P., Robins, A.G.: Wind tunnel measurements of pollutant turbulent fluxes in urban intersections. Atmos. Environ. 46, 669-674 (2012a)

20. Carpentieri, M., Robins, A., Hayden, P., Santi, E.: Mean and turbulent mass flux measurements in an idealised street network. Environ. Pollut. 234, 356-367 (2018). In press

21. Carpentieri, M., Salizzoni, P., Robins, A., Soulhac, L.: Evaluation of a neighbourhood scale, street network dispersion model through comparison with wind tunnel data. Environ. Modell. Softw. 37, 110-124 (2012b)

22. Carruthers, D., Holroyd, R., Hunt, J., Weng, W., Robins, A., Apsley, D., Thompson, D., Smith, F.: UKADMS: A new approach to modelling dispersion in the earth's atmospheric boundary layer. J. Wind Eng. Ind. Aerod. 5, 139-153 (1994)

23. Castro, I.P., Xie, Z.T., Fuka, V., Robins, A.G., Carpentieri, M., Hayden, P., Hertwig, D., Coceal, O.: Measurements and computations of flow in an urban street system. Bound.-Lay. Meteorol. 162(2), 207230 (2017)

24. Chang, J.C., Hanna, S.R.: Air quality model performance evaluation. Meteorol. Atmos. Phys. 87, 167196 (2004)

25. Cimorelli, A.J., Perry, S.G., Venkatram, A., Weil, J.C., Paine, R., Wilson, R.B., Lee, R.F., Peters, W.D., Brode, R.W.: AERMOD: A dispersion model for industrial source applications. Part I: General model formulation and boundary layer characterization. J. Appl. Meteorol. 44(5), 682-693 (2005)

26. Coceal, O., Goulart, E.V., Branford, S., Thomas, T.G., Belcher, S.E.: Flow structure and near-field dispersion in arrays of building-like obstacles. J. Wind Eng. Ind. Aerod. 125, 52-68 (2014)

27. Davidson, M., Snyder, W., Lawson, R., Hunt, J.: Wind tunnel simulations of plume dispersion through groups of obstacles. Atmos. Environ. 30(22), 3715-3731 (1996)

28. Dejoan, A., Santiago, J.L., Martilli, A., Martin, F., Pinelli, A.: Comparison between large-eddy simulation and Reynolds-averaged Navier-Stokes computations for the MUST field experiment. Part II: Effects of incident wind angle deviation on the mean flow and plume dispersion. Bound.-Lay. Meteorol. 135(1), 133-150 (2010)

29. Dobre, A., Arnold, S., Smalley, R., Boddy, J., Barlow, J., Tomlin, A., Belcher, S.: Flow field measurements in the proximity of an urban intersection in London, UK. Atmos. Environ. 39(26), 4647-4657 (2005) 
30. Donnelly, R., Lyons, T., Flassak, T.: Evaluation of results of a numerical simulation of dispersion in an idealised urban area for emergency response modelling. Atmos. Environ. 43(29), 4416-4423 (2009)

31. Fitch, J.P., Raber, E., Imbro, D.R.: Technology challenges in responding to biological or chemical attacks in the civilian sector. Science 302(5649), 1350-1354 (2003)

32. Fuka, V., Xie, Z.T., Castro, I.P., Hayden, P., Carpentieri, M., Robins, A.G.: Scalar fluxes near a tall building in an aligned array of rectangular buildings. Bound.-Lay. Meteorol. (2017). DOI 10.1007/s10546017-0308-4

33. Garbero, V., Salizzoni, P., Soulhac, L.: Experimental study of pollutant dispersion within a network of streets. Bound.-Lay. Meteorol. 136(3), 457-487 (2010)

34. Godschalk, D.: Urban hazard mitigation: creating resilient cities. Nat.1 Hazards Rev. 4, 136-143 (2003)

35. Goulart, E.: Flow and dispersion in urban areas. Ph.D. thesis, University of Reading (2012)

36. Goulart, E., Coceal, O., Belcher, S.: Dispersion of a passive scalar within and above an urban street network. Bound.-Lay. Meteorol. (2017). DOI 10.1007/s10546-017-0315-5

37. Gowardhan, A.A., Pardyjak, E.R., Senocak, I., Brown, M.J.: A CFD-based wind solver for an urban fast response transport and dispersion model. Environ. Fluid Mech. 11(5), 439-464 (2011)

38. Hamlyn, D., Hilderman, T., Britter, R.: A simple network approach to modelling dispersion among large groups of obstacles. Atmos. Environ. 41(28), 5848-5862 (2007)

39. Hanna, S., Baja, E.: A simple urban dispersion model tested with tracer data from Oklahoma City and Manhattan. Atmos. Environ. 43(4), 778-786 (2009)

40. Hanna, S., Chang, J.: Acceptance criteria for urban dispersion model evaluation. Meteorol. Atmos. Phys. 116(3), 133-146 (2012)

41. Hanna, S., White, J., Trolier, J., Vernot, R., Brown, M., Gowardhan, A., Kaplan, H., Alexander, Y., Moussafir, J., Wang, Y., Williamson, C., Hannan, J., Hendrick, E.: Comparisons of JU2003 observations with four diagnostic urban wind flow and Lagrangian particle dispersion models. Atmos. Environ. 45(24), 4073-4081 (2011)

42. Hanna, S.R., Britter, R., Franzese, P.: A baseline urban dispersion model evaluated with Salt Lake City and Los Angeles tracer data. Atmos. Environ. 37(36), 5069-5082 (2003)

43. Hanna, S.R., Britter, R.E.: Wind Flow and Vapor Cloud Dispersion at Industrial and Urban Sites. American Institute of Chemical Engineers, New York (2002)

44. Hanna, S.R., Brown, M.J., Camelli, F.E., Chan, S.T., Coirier, W.J., Kim, S., Hansen, O.R., Huber, A.H., Reynolds, R.M.: Detailed simulations of atmospheric flow and dispersion in downtown Manhattan: An application of five Computational Fluid Dynamics models. B. Am. Meteorol. Soc. 87(12), 1713-1726 (2006)

45. Heist, D.K., Brixey, L.A., Richmond-Bryant, J., Bowker, G.E., Perry, S.G., Wiener, R.W.: The effect of a tall tower on flow and dispersion through a model urban neighborhood Part 1. Flow characteristics. J. Environ. Monit. 11, 2163-2170 (2009)

46. Hertel, O., Berkowicz, R., Larssen, S.: The Operational Street Pollution Model (OSPM). In: H. van Dop, D.G. Steyn (eds.) Air Pollution Modeling and Its Application VIII, pp. 741-750. Springer US, Boston (MA) (1991)

47. Inagaki, M., Kondoh, T., Nagano, Y.: A mixed-time-scale SGS model with fixed model-parameters for practical LES. J. Fluid. Eng. 127, 1-13 (2005)

48. Jackson, P.S.: On the displacement height in the logarithmic velocity profile. J. Fluid Mech. 111, 15-25 (1981)

49. Jones, A., Thomson, D., Hort, M., Devenish, B.: The U.K. Met Office's next-Ggeneration atmospheric dispersion model, NAME III. In: C. Borrego, A.L. Norman (eds.) Air Pollution Modeling and Its Application XVII, pp. 580-589. Springer US (2007)

50. Klein, P., Leitl, B., Schatzmann, M.: Driving physical mechanisms of flow and dispersion in urban canopies. Int. J. Climatol. 27(14), 1887-1907 (2007)

51. McHugh, C., Carruthers, D., Edmunds, H.: ADMS and ADMS-Urban. Int. J. Environ. Pollut. 8(3-6), 438-440 (1997)

52. Nelson, M., Addepalli, B., Hornsby, F., Gowardhan, A., Pardyjak, E., Brown, M.: Improvements to a fastresponse urban wind model. In: 15th Joint Conference on the Applications of Air Pollution Meteorology with the A\&WMA, p. 6. New Orleans, LA (2008)

53. Nelson, M., Brown, M.: The QUIC Start Guide (v6.01). Report LA-UR-13-27291, Los Alamos National Laboratory, Los Alamos (NM), USA (2013)

54. Neophytou, M., Gowardhan, A., Brown, M.: An inter-comparison of three urban wind models using Oklahoma City Joint Urban 2003 wind field measurements. J. Wind Eng. Ind. Aerod. 99(4), 357-368 (2011)

55. Pardyjak, E., Brown, M.: QUIC-URB v.1.1: Theory and Users Guide. Report LA-UR-07-3181, Los Alamos National Laboratory, Los Alamos (NM), USA (2003) 
56. Pullen, J., Boris, J.P., Young, T., Patnaik, G., Iselin, J.: A comparison of contaminant plume statistics from a Gaussian puff and urban CFD model for two large cities. Atmos. Environ. 39(6), 1049-1068 (2005)

57. Riddle, A., Carruthers, D., Sharpe, A., McHugh, C., Stocker, J.: Comparisons between FLUENT and ADMS for atmospheric dispersion modelling. Atmos. Environ. 38(7), 1029-1038 (2004)

58. Robins, A., McHugh, C.: Development and evaluation of the ADMS building effects module. Int. J. Environ. Pollut. 16(1-6), 161-174 (2001)

59. Röckle, R.: Bestimmung der Strömungsverhältnisse im Bereich komplexer Bebauungsstrukturen. Ph.D. thesis, Universität Darmstadt (1990)

60. Sabatino, S.D., Buccolieri, R., Pulvirenti, B., Britter, R.: Simulations of pollutant dispersion within idealised urban-type geometries with CFD and integral models. Atmos. Environ. 41(37), 8316-8329 (2007)

61. Santiago, J.L., Dejoan, A., Martilli, A., Martin, F., Pinelli, A.: omparison between large-eddy simulation and reynolds-averaged navier-stokes computations for the must field experiment. part i: Study of the flow for an incident wind directed perpendicularly to the front array of containers. Bound.-Lay. Meteorol. 135(1), 109-132 (2010)

62. Scire, J.S., Strimaitis, D.G., Yamartino, R.J.: A users guide for the CALPUFF dispersion model. Tech. rep., Earth Tech, Inc., Concord (MA), USA (2000)

63. Settles, G.S.: Fluid mechanics and homeland security. Annual Review of Fluid Mechanics 38(1), 87-110 (2006)

64. Singh, B.: Development of a fast response dispersion model for vitual urban environments. Ph.D. thesis, University of Utah (2012)

65. Singh, B., Hansen, B.S., Brown, M.J., Pardyjak, E.R.: Evaluation of the QUIC-URB fast response urban wind model for a cubical building array and wide building street canyon. Environ. Fluid Mech. 8(4), 281-312 (2008)

66. Singh, B., Pardyjak, E., Norgren, A., Willemsen, P.: Accelerating urban fast response Lagrangian dispersion simulations using inexpensive graphics processor parallelism. Environ. Modell. Softw. 26(6), 739-750 (2011)

67. Soulhac, L.: Modélisation de la dispersion atmosphérique à l'intérieur de la canopée urbaine. Ph.D. thesis, Ecole Centrale de Lyon (2000)

68. Soulhac, L., Garbero, V., Salizzoni, P., Mejean, P., Perkins, R.: Flow and dispersion in street intersections. Atmos. Environ. 43(18), 2981-2996 (2009)

69. Soulhac, L., Lamaison, G., Cierco, F.X., Salem, N.B., Salizzoni, P., Mejean, P., Armand, P., Patryl, L.: SIRANERISK: Modelling dispersion of steady and unsteady pollutant releases in the urban canopy. Atmos. Environ. 140, 242-260 (2016)

70. Soulhac, L., Puel, C., Duclaux, O., Perkins, R.: Simulations of atmospheric pollution in Greater Lyon an example of the use of nested models. Atmos. Environ. 37(37), 5147-5156 (2003)

71. Soulhac, L., Salizzoni, P., Cierco, F.X., Perkins, R.: The model SIRANE for atmospheric urban pollutant dispersion; part I, presentation of the model. Atmos. Environ. 45, 7379-7395 (2011)

72. Soulhac, L., Salizzoni, P., Mejean, P., Didier, D., Rios, I.: The model SIRANE for atmospheric urban pollutant dispersion; part II, validation of the model on a real case study. Atmos. Environ. 49, 320-337 (2012)

73. Soulhac, L., Salizzoni, P., Mejean, P., Perkins, R.: Parametric laws to model urban pollutant dispersion with a street network approach. Atmos. Environ. 67, 229-241 (2013)

74. Stein, A.F., Draxler, R.R., Rolph, G.D., Stunder, B.J.B., Cohen, M.D., Ngan, F.: NOAAs HYSPLIT Atmospheric Transport and Dispersion Modeling System. B. Am. Meteorol. Soc. 96(12), 2059-2077 (2015)

75. Sykes, R.I., Henn, D.S., Parker, S.F.: SCIPUFF-A generalized hazard dispersion model. In: Preprints, Ninth Joint Conference on Applications of Air Pollution Meteorology with AWMA, Amer. Meteor. Soc., pp. 184-188. Atlanta, GA (1996)

76. Taylor, K.E.: Summarizing multiple aspects of model performance in a single diagram. J. Geophys. Res.-Atmos. 106(D7), 7183-7192 (2001)

77. Thykier-Nielsen, S., Deme, S., Mikkelsen, T.: Description of the Atmospheric Dispersion Module RIMPUFF. Tech. Rep. RODOS(WG2)-TN(98)-02, Risø National Laboratory, Roskilde, Denmark (1999)

78. Tinarelli, G., Brusasca, G., Oldrini, O., Anfossi, D., Castelli, S.T., Moussafir, J.: Micro-Swift-Spray (MSS): A new modelling system for the simulation of dispersion at microscale. General description and validation. In: C. Borrego, A.L. Norman (eds.) Air Pollution Modeling and Its Application XVII, pp. 449-458. Springer US, Boston, MA (2007)

79. Tominaga, Y., Stathopoulos, T.: CFD simulation of near-field pollutant dispersion in the urban environment: A review of current modeling techniques. Atmos. Environ. 79, 716-730 (2013)

80. Tominaga, Y., Stathopoulos, T.: Ten questions concerning modeling of near-field pollutant dispersion in the built environment. Build. Environ. 105, 390-402 (2016) 
81. Williams, M., Brown, M., Singh, B., Boswell, D.: QUIC-PLUME Theory Guide. Tech. Rep. LA-UR2004, 04-0561, Los Alamos National Laboratory, Los Alamos (NM), USA (2004)

82. Wood, C.R., Barlow, J.F., Belcher, S.E., Dobre, A., Arnold, S.J., Balogun, A.A., Lingard, J.J.N., Smalley, R.J., Tate, J.E., Tomlin, A.S., Britter, R.E., Cheng, H., Martin, D., Petersson, F.K., Shallcross, D.E., White, I.R., Neophytou, M.K., Robins, A.G.: Dispersion experiments in central London: The 2007 DAPPLE project. B. Am. Meteorol. Soc. 90(7), 955-969 (2009) 